\title{
A Multiple Attribute Decision Making Approach Based on New Similarity Measures of Interval-valued Hesitant Fuzzy Sets
}

\author{
Yi Liu ${ }^{1,2,3 *}$, Jun Liu ${ }^{3}$, Zhiyong Hong ${ }^{4}$ \\ ${ }^{1}$ Data Recovery Key Lab of Sichuan Province, Neijiang Normal University, \\ Neijiang 641000, Sichuan, P.R. China \\ 2 School of Mathematics and Information Science, Neijiang Normal University, \\ Neijiang 641000, Sichuan, P.R. China \\ E-mail: liuyiyl@126.com \\ ${ }^{3}$ School of Computing and Mathematics, Ulster University \\ Jordanstown Campus, Northern Ireland BT 37 OTR, UK \\ E-mail:J.liu@ulster.ac.uk \\ ${ }^{4}$ School of Computer Science, Wuyi University \\ Jiangmen 529020, Guangdong, P.R. China \\ E-mail: hongmr@163.com
}

Received 15 June 2017

Accepted 14 September 2017

\begin{abstract}
Hesitant fuzzy sets, as an extension of fuzzy sets to deal with uncertainty, have attracted much attention since its introduction, in both theory and application aspects. The present work is focused on the interval-valued hesitant fuzzy sets (IVHFSs) to manage additional uncertainty. Now that distance and similarity as a kind of information measures are essential and important numerical indexes in fuzzy set theory and all their extensions, the present work aims at investigating distance and similarity measures in the IVHFSs and then employing them into multiple attribute decision making application. To begin with, II-type generalized interval-valued hesitant fuzzy distance is firstly introduced in the IVHFS, along with its properties and its relationships with the traditional Hamming-Distance and the Euclidean distance. Afterwards, another interval-valued hesitant fuzzy $L p$ distance based on $L p$ metric is proposed and its relationship with the Hausdorff distance is discussed. In addition, different from most of similarity measures with dependent on the corresponding distances, a new similarity measure based on set-theoretic approach for IVHFSs is introduced and its properties are discussed; especially, a relative similarity measure is proposed based on the positive ideal IVHFS and the negative ideal IVHFS. Finally, we describe how the IVHFS and its relative similarity measure can be applied to multiple attribute decision making. A numerical example is then provided to illustrate the effectiveness of the proposed method.
\end{abstract}

Keywords: Interval-valued hesitant fuzzy set, II-type interval-valued hesitant fuzzy distance, intervalvalued hesitant fuzzy $L_{p}$ distance, relative similarity measure, multiple attribute decision making.

\footnotetext{
* Email:liuyiyl@126.com.
} 


\section{Introduction}

Fuzzy set, a generalization of classical set theory introduced by Zadeh ${ }^{23}$, has drawn the attention of many researchers who have extended the fuzzy sets to intuitionistic fuzzy sets (IFSs) ${ }^{2}$, intervalvalued intuitionistic fuzzy sets (IVIFSs) ${ }^{1}$, intervalvalue Pythagorean fuzzy set ${ }^{15}$ and so on, which are also applied to some decision making ${ }^{11}$. In 2010 , Torra ${ }^{20}$ introduced an important extension of fuzzy sets named the hesitant fuzzy sets (HFSs) which permit the membership degree of an element to a set to be represented as several possible values between 0 and 1, human beings hesitate among a set of membership degrees and they need to represent such a hesitation. Rodriguez ${ }^{17,18}$ recently provided a position and perspective analysis of HFSs in decision making, which gave a detailed review on HFS and its application in decision making, especially pointed out some important challenges.

In many real world decision making problems, due to insufficiency in available information, it may be difficult for decision makers to exactly quantify their opinions with a crisp number, instead they may prefer an interval number within $[0,1]$. Accordingly, Chen ${ }^{5,6}$ introduced the concept of interval-valued hesitant fuzzy sets (IVHFSs), which represent the membership degrees of an element to a set with several possible interval values.

Information measures (specially, distance, similarity, and entropy) for IVHFSs have played the key roles in the development of the IVHFS theory and its applications. IVHFS distance and similarity measures indicate the distance and similarity degrees of two IVHFSs, and the entropy of an IVHFS describes the fuzziness degree of an IVHFS. Therefore, many researchers have investigated these three concepts for IVHFSs from different points of views. For example, Farhadinia ${ }^{7}$ investigated the relationship among the entropy, the similarity and the distance for IVHFSs and applied the similarity measures to two clustering algorithms with applications in data analysis and classification. He also investigated the approach for deriving the correlation coefficient of dual IVHFSs ${ }^{8}$ and proposed division and subtraction formulas for IVHFSs ${ }^{9}$. Gitinavard 10 proposed a decision model based on IVHFSs using the distance measures and applied it to the industrial selection problems. Jin ${ }^{13}$ proposed a multiple attribute group decision making method on the basis of some information measures under the intervalvalued hesitant fuzzy environment, where the axiomatic definitions of continuous entropy and continuous similarity measures on IVHFSs were introduced and the method was applied to emergency risk assessment. Quiros ${ }^{16}$ studied entropy measures under interval-valued hesitant fuzzy environment and built the entropy measure using three different measures: fuzziness, lack of knowledge and hesitance. Wei 22 proposed a variety of distance measures for IVHFSs, based on which the corresponding similarity measures are derived, and some properties of these distance measures and similarity measures were investigated, but the general definition of distance measures and similarity measure are not given like the notions of fuzzy sets. Farhadini ${ }^{9}$ gave the axiom definition of distance measures of HFSs and IVHFSs, and all distance must be in $[0,1]$, however, there are many distance measures beyond $[0,1]$. So we modify the axiom definitions of distance and similarity measures for IVHFSs and propose some new distance and similarity measures between IVHFSs, the new distance measures are more reasonable since they are very similar with the existing ones except for some minor modification of the expression. Wei ${ }^{22}$ introduced a generalized interval-valued hesitant Hamming-Hausdorff distance which is a generalization of interval-valued hesitant normal Hamming-Hausdorff distance, we will introduce new distance measure whose limit is the interval-valued hesitant normal HammingHausdorff distance from the point of view of $L_{p}$ metric. They are another new reasonable distance measures from different point of view and the main advantages of the new distance measures is that they do not need to be normalized and more reasonable since they are very similar with the existing ones except for some minor modification of the expression.

The IVHFSs method and its distance measures were applied to show potential evaluation of emerging technology commercialization with intervalvalued hesitant fuzzy information.

As pointed out in ${ }^{17}$, one of the great challenges 
is to further develop foundation in the HFS theory, e.g., the notion of entropy, similarity and distance measures. Based on the existing work as reviewed above about the information measures for IVHFSs, in the present work, we continue investigating distance and similarity measures for IVHFSs from different points of views. The main contributions are summarized as below: (1) introduce a new generalized interval-valued hesitant fuzzy distance, named II-type generalized interval-valued hesitant fuzzy distance which is a generalization of the famous Haming-distance and the Euclidean distance; (2) introduce an interval-valued hesitant fuzzy $L p$ distance based on $L_{p}$ metric, and shows that the Hausdorff distance of two IVHFSs is the limit of $L_{p}$ distance when $p \rightarrow+\infty$; (3) propose a new similarity measure of IVHFSs based on the set theoretical view after analysing and summarizing the existing similarity measures which are dependent on the distance measures; (4) propose a new relative information measure, that is, a new relative similarity measure based on the positive ideal IVHFS and the negative ideal IVHFS; and 5) propose a multiple attributes decision making method under IVHFSs based on the new relative similarity measure.

The rest of the paper is organized as follows. In Section 2, we review some definitions on IVHFSs, some existing distance and similarity measures of IVHFSs, which are used in the analysis throughout this paper. Section 3 is devoted to the main results concerning the distances of IVHFSs: 1) II-type generalized interval-valued hesitant fuzzy distance; 2 ) the interval-valued hesitant fuzzy $L p$ distance based on $L_{p}$ metric. Section 4 is focused on the new similarity measures base on set theory after analysing the existing similarity measures and proposing a generic definition of a family of similarity measures, which are dependent on the distance measures. In Section 5 , we propose the concept of relative similarity measure of IVHFSs and construct multiple attribute decision-making approach based on the relative similarity measure. Consequently, a practical example is provided in Section 6 to illustrate this method and compare the proposed methods with the existing one in Wei2013. This paper is concluded in Section 7.

\section{Preliminaries}

Necessary basic concepts to understand the definition of distance and similarity measures proposed in this work are given in this section. It has been split into two subsections. Basic concepts about the interval-valued hesitant fuzzy set are explained in the first subsection. In the second subsection, the axiomatic definitions of distance and similarity measures for IVHFSs are given, which establish foundations for the follow-up development of this work in other sections.

\subsection{Interval-Valued Hesitant Fuzzy Set}

Definition 1. ${ }^{20}$ Let $X=\left\{x_{1}, x_{2}, \cdots, x_{n}\right\}$ be a reference set. A hesitant fuzzy set (HFS) $F$ on $X$ is defined in terms of a function $h_{F}(x)$ that returns a subset of $[0,1]$ when it is applied to $X$, i.e., $F=\left\{\left\langle x, h_{F}(x) \mid x \in X\right\rangle\right\}$ where $h_{F}(x)$ is a set of some different values in $[0,1]$, representing the possible membership degrees of the element $x \in X$ to $F$. $h_{F}(x)$ is called a hesitant fuzzy element (HFE), a basic unit of HFS.

Definition 2. ${ }^{5,6}$ Let $X=\left\{x_{1}, x_{2}, \cdots, x_{n}\right\}$ be a reference set. An interval-valued hesitant fuzzy set (IVHFS) on $X$ is

$$
\widetilde{F}=\left\{\left\langle x, h_{\widetilde{F}}(x)\right\rangle \mid x \in X\right\},
$$

where $h_{\widetilde{F}}(x)$ denotes all possible interval-valued membership degrees of the element $x \in X$ to the set $\widetilde{F}$. For convenience, we call $h_{\widetilde{F}}(x)$ an intervalvalued hesitant fuzzy element (IVHFE), which reads

$$
h_{\widetilde{F}}(x)=\left\{\widetilde{r} \mid \widetilde{r} \in h_{\widetilde{F}}(x)\right\} .
$$

Here $\widetilde{r}$ is an interval number. An IVHFE is the basic unit of an IVHFS, and it can be considered as a special case of the IVHFS. The relationship between IVHFE and IVHFS is similar to that between interval-valued fuzzy number and interval-valued fuzzy set.

Example 1. If $X=\left\{x_{1}, x_{2}, x_{3}\right\}$ is a reference set, $h_{F}\left(x_{1}\right)=\{0.1,0.2\}, h_{F}\left(x_{2}\right)=\{0.4,0.5,0.7\}$, and $h_{F}\left(x_{3}\right)=\{0.2\}$ are the possible membership degrees of $x_{i}(i=1,2,3)$ to the set $F$, then $F$ can be regarded 
as a HFS and is presented as follows:

$$
\begin{aligned}
F=\{\quad & \left\langle x_{1},\{0.1,0.2\}\right\rangle,\left\langle x_{2},\{0.4,0.5,0.7\}\right\rangle, \\
& \left.\left\langle x_{3},\{0.2\}\right\rangle\right\} .
\end{aligned}
$$

For the reference set $X=\left\{x_{1}, x_{2}, x_{3}\right\}$, if $h_{\widetilde{F}}\left(x_{1}\right)=$ $\{[0.1,0.2]\}, \quad h_{\widetilde{F}}\left(x_{2}\right)=\{[0.5,0.7],[0.3,0.4]\}$, $h_{\widetilde{F}}\left(x_{3}\right)=\{[0.2,0.5]\}$ are the possible membership degrees of $x_{i}(i=1,2,3)$ to the set $\widetilde{F}$. then $\widetilde{F}$ can be regarded as an IVHFS and is presented as follows:

$$
\begin{array}{r}
\widetilde{F}=\left\{\left\langle x_{1},\{[0.1,0.2]\}\right\rangle,\left\langle x_{2},\{[0.5,0.7],[0.3,0.4]\}\right\rangle,\right. \\
\left.\left\langle x_{3},\{[0.2,0.5]\}\right\rangle\right\} .
\end{array}
$$

Hereafter, for the notation convenience, a real number $a$ can be regarded as an interval $[a, a]$, so it is also regarded as a special IVHFS, that is, $\langle x,\{[a, a]\}\rangle$.

Remark 1. From Definition 2.2, it should be noted that HFSs permit the membership of an element to be a set of several possible values. All these possible values are crisp real numbers that belong to $[0$, 1]. However, IVHFSs permit the membership degrees of certain elements to a set with several possible interval values. Therefore, IVHFSs are more reasonable for experts to assign their evaluation values in some practical problems. Whist we can see that both HFSs and IVFSs are all particular cases of IVHFSs.

We use $\mathscr{I} \mathscr{V} \mathscr{H} \mathscr{F} \mathscr{S}(X)$ to denote the class of all IVHFSs of a reference set $X$. In addition, for an IVHFE $h_{\widetilde{F}}\left(x_{i}\right)$, it is necessary to arrange the intervals in an increasing order. To achieve the goal, the score function and accuracy function, given in ${ }^{16}$, are employed to compare two intervals and described as follows:

Let $X=\left[x_{1}, x_{2}\right]$, the score function of $X$ defined as $S(X)=x_{2}-x_{1}$ and accuracy function of $X$ defined as $H(X)=x_{1}+x_{2}$. Let $A=\left[a_{1}, b_{1}\right]$ and $B=\left[a_{2}, b_{2}\right]$ be two intervals, then

$$
A \leqslant B \Longleftrightarrow\left\{\begin{array}{l}
H(A) \leqslant H(B) \\
\text { or } \\
H(A)=H(B) \text { and } S(A) \leqslant S(B) .
\end{array}\right.
$$

Let $h_{\widetilde{F}}^{\sigma(j)}\left(x_{i}\right)$ stand for the $j$ th smallest interval in the $h_{\widetilde{F}}\left(x_{i}\right)$, where

$$
\begin{aligned}
& h_{\widetilde{F}}^{\sigma(j)}\left(x_{i}\right)=\left[h_{\widetilde{F}}^{\sigma(j) L}\left(x_{i}\right), h_{\widetilde{F}}^{\sigma(j) U}\left(x_{i}\right)\right] . \\
& h_{\widetilde{F}}^{\sigma(j) L}\left(x_{i}\right)=\inf _{j}\left(h_{\widetilde{F}}^{\sigma(j)}\left(x_{i}\right)\right), \\
& h_{\widetilde{F}}^{\sigma(j) U}\left(x_{i}\right)=\sup _{j}\left(h_{\widetilde{F}}^{\sigma(j)}\left(x_{i}\right)\right), \\
& \quad 1 \leqslant i \leqslant n, 1 \leqslant j \leqslant n\left(h_{\widetilde{F}}\left(x_{i}\right)\right) .
\end{aligned}
$$

Here $n\left(h_{\widetilde{F}}\left(x_{i}\right)\right)$ denotes the number of intervals in the $h_{\widetilde{F}}\left(x_{i}\right)$.

Example 2. Let $X=\left\{x_{1}, x_{2}\right\}$ be a reference set. Define an IVHFS $\widetilde{F}$ on $X$ as follows:

$$
\begin{array}{r}
\widetilde{F}=\left\{\left\langle x_{1},\{[0.5,0.6],[0.2,0.3],[0.4,0.6]\}\right\rangle,\right. \\
\left.\left\langle x_{2},\{[0.3,0.5],[0.4,0.6]\}\right\rangle\right\} .
\end{array}
$$

Then $h_{\widetilde{F}}^{\sigma(1)}\left(x_{1}\right)=[0.2,0.3], h_{\widetilde{F}}^{\sigma(2)}\left(x_{1}\right)=[0.4,0.6]$, $h_{\widetilde{F}}^{\sigma(3)}\left(x_{1}\right)=[0.5,0.6], \quad h_{\widetilde{F}}^{\sigma(1)}\left(x_{2}\right)=[0.3,0.5]$, $h_{\widetilde{F}}^{\sigma(2)}\left(x_{2}\right)=[0.4,0.6]$.

It follows from Example 1 and Example 2 that the number of intervals in different IVHFEs may be different. We use $n\left(h_{\widetilde{F}}(x)\right)$ to stand for the number of intervals in the IVHFE $h_{\widetilde{F}}(x)$. For any two IVHFSs $\widetilde{A}, \widetilde{B}$ on the same reference set $X$, set $n_{x}(\widetilde{A}, \widetilde{B})=\max \left\{n\left(h_{\widetilde{A}}(x)\right), n\left(h_{\widetilde{B}}(x)\right)\right\}$ for some $x \in X$. In order to compare two IVHFSs conveniently and correctly, the two IVHFEs $h_{\widetilde{A}}(x)$ and $h_{\widetilde{B}}(x)$ should have the same number of intervals, that is, $n_{x}(\widetilde{A}, \widetilde{B})$. Therefore, if there are less intervals in $h_{\widetilde{A}}(x)$ than in $h_{\widetilde{B}}(x)$, an extension of $h_{\widetilde{A}}(x)$ should be considered optimistically by repeating its maximum interval until it has the same number of the intervals as that in $h_{\widetilde{B}}(x)$, that is $n_{x}(\widetilde{A}, \widetilde{B})$, in short denoted as $n_{x}($ of course, an extension of $h_{\widetilde{A}}(x)$ is also considered pessimistically by repeating its minimal interval until it has the same number of the intervals as that in $h_{\widetilde{B}}(x)$. Although the results may be different if we extend the shorter one by adding different values, this is reasonable because the decision makers risk preferences can directly influence the final decision. In this paper, we assume that the decision makers are all optimistic (other situations can be studied similarly)). In the subsequent section, without loss of generality, assume that the IVHFEs involved in 
distance or similarity measures have the same number of intervals.

Example 3. Let $X=\left\{x_{1}, x_{2}\right\}$ be a reference set. Define two IVHFSs $\widetilde{F}$ and $\widetilde{G}$ on $X$ as follows:

$$
\begin{array}{r}
\widetilde{F}=\left\{\left\langle x_{1},\{[0.5,0.6],[0.2,0.3],[0.4,0.6]\}\right\rangle,\right. \\
\left.\left\langle x_{2},\{[0.3,0.5],[0.4,0.6]\}\right\rangle\right\} ; \\
\widetilde{G}=\left\{\left\langle x_{1},\{[0.4,0.5],[0.5,0.6]\}\right\rangle,\right. \\
\left.\left\langle x_{2},\{[0.3,0.5],[0.4,0.6],[0.6,0.7]\}\right\rangle\right\} .
\end{array}
$$

Then, to keep the same number of intervals, after the extensions on both $\widetilde{F}$ and $\widetilde{G}$ respectively, these two IVHFSs can be re-expressed respectively as follows:

$$
\begin{aligned}
& \widetilde{F}=\left\{\left\langle x_{1},\{[0.2,0.3],[0.4,0.6],[0.5,0.6]\}\right\rangle,\right. \\
&\left.\left\langle x_{2},\{[0.3,0.5],[0.4,0.6],[0.4,0.6]\}\right\rangle\right\} ; \\
& \widetilde{G}=\left\{\left\langle x_{1},\{[0.4,0.5],[0.5,0.6],[0.5,0.6]\}\right\rangle,\right. \\
&\left.\left\langle x_{2},\{[0.3,0.5],[0.4,0.6],[0.6,0.7]\}\right\rangle\right\} .
\end{aligned}
$$

Definition 3 . $^{7}$ Let $X$ be a reference set, $\widetilde{F}$ and $\widetilde{G}$ be two IVHFSs on $X$. Then, one kind of ordering for IVHFSs, i.e., the component-wise ordering of IVHFSs, is defined as follows:

$$
\begin{aligned}
\widetilde{F} \leqslant \widetilde{G} \Leftrightarrow & h_{\widetilde{F}}^{\sigma(j) L}\left(x_{i}\right) \leqslant h_{\widetilde{G}}^{\sigma(j) L}\left(x_{i}\right), \\
& h_{\widetilde{F}}^{\sigma(j) U}\left(x_{i}\right) \leqslant h_{\widetilde{G}}^{\sigma(j) U}\left(x_{i}\right),
\end{aligned}
$$

where $x_{i} \in X, 1 \leqslant i \leqslant n, 1 \leqslant j \leqslant n_{x_{i}}$.

\subsection{Axiomatic Definitions of Distance and Similarity Measures for IVHFSs}

Distance and similarity measures are the fundamental and important issues of theory of fuzzy sets. The axiomatic definitions of distance and similarity measure between two IVHFSs is introduced in ${ }^{7}$ and reviewed as below in Def. 4 and Def. 5 respectively.

Definition $4 .{ }^{7}$ Let $\widetilde{F}$ and $\widetilde{G}$ be IVHFSs on the reference set $X=\left\{x_{1}, x_{2}, \cdots, x_{n}\right\}$ and $d$ a real function: $\mathscr{I} \mathscr{V} \mathscr{H} \mathscr{F} \mathscr{S} \times \mathscr{I} \mathscr{V} \mathscr{H} \mathscr{F} \mathscr{S} \rightarrow[0,1] . d$ is a distance measure between $\widetilde{F}$ and $\widetilde{G}$ if $d$ satisfies the following properties:

(D1) $0 \leqslant d(\widetilde{F}, \widetilde{G}) \leqslant 1$;

(D2) $d(\widetilde{F}, \widetilde{G})=0 \Leftrightarrow \widetilde{F}=\widetilde{G}$;

(D3) $d(\widetilde{F}, \widetilde{G})=d(\widetilde{G}, \widetilde{F})$;

(D4) $d\left(\widetilde{F}, \widetilde{F}^{C}\right)=1$ if and only if $\widetilde{F}=$ $\{\langle x,\{[0,0]\}\rangle\}$ or $\widetilde{F}=\{\langle x,\{[1,1]\}\rangle\}$;

(D5) let $\widetilde{E}$ be an IVHFS and $\widetilde{E} \leqslant \widetilde{F} \leqslant \widetilde{G}$. Then $d(\widetilde{E}, \widetilde{F}) \leqslant d(\widetilde{E}, \widetilde{G})$ and $d(\widetilde{F}, \widetilde{G}) \leqslant d(\widetilde{E}, \widetilde{G})$. Here in (D4), $\left.\widetilde{F}^{C}=\left\{\left\langle x, \cup_{\widetilde{r} \in h_{\widetilde{M}(x)}}\left\{\widetilde{r}^{C}\right\}\right\}\right\rangle \mid x \in X\right\}$ denotes the complement of $\widetilde{F}$, and $\widetilde{r}^{C}$ is the complement of an interval $\widetilde{r}$.

Moreover, various distance measures of two IVHFSs $\widetilde{F}, \widetilde{G}$ are proposed in 7,21 . For example, given two IVHFSs $\widetilde{F}, \tilde{G}$, $h_{\widetilde{F}}^{\sigma(j)}\left(x_{i}\right)=\left[h_{\widetilde{F}}^{\sigma(j) L}\left(x_{i}\right), h_{\widetilde{F}}^{\sigma(j) U}\left(x_{i}\right)\right]$ and $h_{\widetilde{G}}^{\sigma(j)}\left(x_{i}\right)=$ $\left[h_{\widetilde{G}}^{\sigma(j) L}\left(x_{i}\right), h_{\widetilde{G}}^{\sigma(j) U}\left(x_{i}\right)\right]$ denote the $j$ th smallest intervals in $h_{\widetilde{F}}\left(x_{i}\right)$ and $h_{\widetilde{G}}\left(x_{i}\right)$, respectively.

The interval-valued hesitant fuzzy Hamming distance is defined in ${ }^{7}$ as follows:

$$
d_{1}(\widetilde{F}, \widetilde{G})=\frac{1}{n} \sum_{i=1}^{n}\left[\frac{1}{2 n_{x_{i}}} \sum_{j=1}^{n_{x_{i}}}\left(\left|h_{\widetilde{F}}^{\sigma(j) L}\left(x_{i}\right)-h_{\widetilde{G}}^{\sigma(j) L}\left(x_{i}\right)\right|+\left|h_{\widetilde{F}}^{\sigma(j) U}\left(x_{i}\right)-h_{\widetilde{G}}^{\sigma(j) U}\left(x_{i}\right)\right|\right)\right] .
$$

The interval-valued hesitant fuzzy Euclidean dis-

tance is defined in ${ }^{21}$ as follows:

$$
d_{2}(\widetilde{F}, \widetilde{G})=\sqrt{\frac{1}{n} \sum_{i=1}^{n}\left[\frac{1}{2 n_{x_{i}}} \sum_{j=1}^{n_{x_{i}}}\left(\left|h_{\widetilde{F}}^{\sigma(j) L}\left(x_{i}\right)-h_{\widetilde{G}}^{\sigma(j) L}\left(x_{i}\right)\right|^{2}+\left|h_{\widetilde{F}}^{\sigma(j) U}\left(x_{i}\right)-h_{\widetilde{G}}^{\sigma(j) U}\left(x_{i}\right)\right|^{2}\right)\right]} .
$$


The generalized interval-valued hesitant fuzzy

distance is defined in ${ }^{21}$ as follows:

$$
d_{3}(\widetilde{F}, \widetilde{G})=\left\{\frac{1}{n} \sum_{i=1}^{n}\left[\frac{1}{2 n_{x_{i}}} \sum_{j=1}^{n_{x_{i}}}\left(\left|h_{\widetilde{F}}^{\sigma(j) L}\left(x_{i}\right)-h_{\widetilde{G}}^{\sigma(j) L}\left(x_{i}\right)\right|^{p}+\left|h_{\widetilde{F}}^{\sigma(j) U}\left(x_{i}\right)-h_{\widetilde{G}}^{\sigma(j) U}\left(x_{i}\right)\right|\right)^{p}\right]\right\}^{\frac{1}{p}}, p>0 .
$$

We can see from the above distances that $d_{i}(\widetilde{F}, \widetilde{G})(i=1,2,3)$ all satisfy $0 \leqslant d_{i}(\widetilde{F}, \widetilde{G}) \leqslant 1$,

sometimes some defined operators satisfy $(D 2)-$ $(D 5)$, but did not satisfy $\left(D_{1}\right)$. For example,

$$
d_{1}^{\prime}(\widetilde{F}, \widetilde{G})=\frac{1}{n} \sum_{i=1}^{n}\left[\frac{1}{n_{x_{i}}} \sum_{j=1}^{n_{x_{i}}}\left(\left|h_{\widetilde{F}}^{\sigma(j) L}\left(x_{i}\right)-h_{\widetilde{G}}^{\sigma(j) L}\left(x_{i}\right)\right|+\left|h_{\widetilde{F}}^{\sigma(j) U}\left(x_{i}\right)-h_{\widetilde{G}}^{\sigma(j) U}\left(x_{i}\right)\right|\right)\right]
$$

Obviously, $d_{1}^{\prime}(\widetilde{F}, \widetilde{G}) \leqslant 2$, which shows that $d_{1}^{\prime}(\widetilde{F}, \widetilde{G})$ does not satisfy the condition $(D 1)$. In order to distinguish it from Def. 6 introduced in the subsequent section, $d(\widetilde{F}, \widetilde{G})$ in Def. 4 is also called an interval-valued hesitant fuzzy normalized distance between two IVHFSs $\widetilde{F}$ and $\widetilde{G}$.

Therefore, $\quad d_{i}(\widetilde{F}, \widetilde{G})(i=1,2,3)$ are called interval-valued hesitant fuzzy normalized Hamming distance, interval-valued hesitant fuzzy normalized Euclidean distance and generalized interval-valued hesitant fuzzy normalized distance, respectively.

Definition 5. ${ }^{7}$. Let $\widetilde{F}$ and $\widetilde{G}$ be IVHFSs on the reference set $X=\left\{x_{1}, x_{2}, \cdots, x_{n}\right\}$ and $s$ a real function: $\mathscr{I} \mathscr{V} \mathscr{H} \mathscr{F} \mathscr{S} \times \mathscr{I} \mathscr{V} \mathscr{H} \mathscr{F} \mathscr{S} \rightarrow[0,1] . s$ is called a similarity measure between $\widetilde{F}$ and $\widetilde{G}$ if $s$ satisfies the following properties:

(S1) $0 \leqslant s(\widetilde{F}, \widetilde{G}) \leqslant 1$;

(S2) $s(\widetilde{F}, \widetilde{G})=1 \Leftrightarrow \widetilde{F}=\widetilde{G}$;

(S3) $s(\widetilde{F}, \widetilde{G})=s(\widetilde{G}, \widetilde{F})$;

(S4) if $\widetilde{F}=\{\langle x,\{[0,0]\}\rangle\}$ or $\widetilde{F}=\{\langle x,\{[1,1]\}\rangle\}$, then $s\left(\widetilde{F}, \widetilde{F}^{C}\right)=0$;

(S5) let $\widetilde{E}$ be an IVHFS and $\widetilde{E} \leqslant \widetilde{F} \leqslant \widetilde{G}$. Then $s(\widetilde{E}, \widetilde{F}) \geqslant s(\widetilde{E}, \widetilde{G})$ and $s(\widetilde{F}, \widetilde{G}) \geqslant s(\widetilde{E}, \widetilde{G})$. Here, $\left.\widetilde{F}^{C}=\left\{\left\langle x, \cup_{\widetilde{r} \in h_{\widetilde{M}(x)}}\left\{\widetilde{r}^{C}\right\}\right\}\right\rangle \mid x \in X\right\}$, as the same as defined above. .

\section{New Distance Measures for IVHFSs}

In this section, we will introduce two new distance measures between two IVHFSs, that is, IItype generalized interval-valued hesitant fuzzy distance and the interval-valued hesitant fuzzy $L_{p}$ distance based on $L_{p}$ metric, along with their properties. They are another new reasonable distance measures from different point of view and the main advantages of the new distance measures is that they do not need to be normalized and more reasonable since they are very similar with the existing ones except for some minor modification of the expression. If not otherwise specified, in the subsequent sections, we always assume that $\widetilde{F}, \widetilde{G}$ are two IVHFSs and $h_{\widetilde{F}}^{\sigma(j)}\left(x_{i}\right)=\left[h_{\widetilde{F}}^{\sigma(j) L}\left(x_{i}\right), h_{\widetilde{F}}^{\sigma(j) U}\left(x_{i}\right)\right]$ and $h_{\widetilde{G}}^{\sigma(j)}\left(x_{i}\right)=\left[h_{\widetilde{G}}^{\sigma(j) L}\left(x_{i}\right), h_{\widetilde{G}}^{\sigma(j) U}\left(x_{i}\right)\right]$ denote the $j$ th smallest intervals in $h_{\widetilde{F}}\left(x_{i}\right)$ and $h_{\widetilde{G}}\left(x_{i}\right)$, respectively.

\subsection{II-type generalized interval-valued hesitant fuzzy distance}

We have listed three kinds of important distances in Section 2. Obviously, if $p=1, p=2$ in Eq.(3), then $d_{3}(\widetilde{F}, \widetilde{G})$ will be degenerated to $d_{1}(\widetilde{F}, \widetilde{G})$ and $d_{2}(\widetilde{F}, \widetilde{G})$ respectively, which reflects the connection between these two sets of distances. From $d_{1}(\widetilde{F}, \widetilde{G})$, assume

$$
d_{i}=\frac{1}{2 n_{x_{i}}} \sum_{j=1}^{n_{x_{i}}}\left(\left|h_{\widetilde{F}}^{\sigma(j) L}\left(x_{i}\right)-h_{\widetilde{G}}^{\sigma(j) L}\left(x_{i}\right)\right|+\left|h_{\widetilde{F}}^{\sigma(j) U}\left(x_{i}\right)-h_{\widetilde{G}}^{\sigma(j) U}\left(x_{i}\right)\right|\right) .
$$


It is actually the distance between the $i$ th IVHFEs of $\widetilde{F}$ and $\widetilde{G}$. However, $d_{1}(\widetilde{F}, \widetilde{G})$ is the distances between all IVHFEs of $\widetilde{F}$ and $\widetilde{G}$. From this point of view, we can modify $d_{3}(\widetilde{F}, \widetilde{G})$ and redefine another generalized measure $d_{4}(\widetilde{F}, \widetilde{G})$ between $\widetilde{F}$ and $\widetilde{G}$ as follows:

$$
d_{4}(\widetilde{F}, \widetilde{G})=\frac{1}{n} \sum_{i=1}^{n}\left[\frac{1}{2 n_{x_{i}}} \sum_{j=1}^{n_{x_{i}}}\left(\left|h_{\widetilde{F}}^{\sigma(j) L}\left(x_{i}\right)-h_{\widetilde{G}}^{\sigma(j) L}\left(x_{i}\right)\right|^{p}+\left|h_{\widetilde{F}}^{\sigma(j) U}\left(x_{i}\right)-h_{\widetilde{G}}^{\sigma(j) U}\left(x_{i}\right)\right|^{p}\right)\right]^{\frac{1}{p}}, p>0 .
$$

If $p=1$ in Eq. (5), Eq. (5) is also degenerated to Eq. (1). If $p=2$ in Eq. (5), Eq. (5) will not be degenerated to Eq. (2), instead, it is degenerated to another measure $d_{5}(\widetilde{F}, \widetilde{G})$ between $\widetilde{F}$ and $\widetilde{G}$ as follows:

$$
d_{5}(\widetilde{F}, \widetilde{G})=\frac{1}{n} \sum_{i=1}^{n} \sqrt{\frac{1}{2 n_{x_{i}}} \sum_{j=1}^{n_{x_{i}}}\left(\left|h_{\widetilde{F}}^{\sigma(j) L}\left(x_{i}\right)-h_{\widetilde{G}}^{\sigma(j) L}\left(x_{i}\right)\right|^{2}+\left|h_{\widetilde{F}}^{\sigma(j) U}\left(x_{i}\right)-h_{\widetilde{G}}^{\sigma(j) U}\left(x_{i}\right)\right|^{2}\right)} .
$$

Of course, it is natural to consider the rationality of Eq. (5) in terms of distance measure. In other words, we need to check if $d_{4}(\widetilde{F}, \widetilde{G})$ satisfies (D1)(D5) in Def. 4 or not. The answer is positive and stated in the following theorem.

Theorem 1. $d_{4}(\widetilde{F}, \widetilde{G})$ is an interval-valued hesitant fuzzy normalized distance between two IVHFSs, $\widetilde{F}$ and $\widetilde{G}$.

Proof. Obviously, it is straightforward to see that $d_{4}(\widetilde{F}, \widetilde{G})$ satisfies (D1), (D2), (D3) and (D4) in Definition 4. Now, we consider the axiom (D5). Let $\widetilde{E} \leqslant$ $\widetilde{F} \leqslant \widetilde{G}$. Then $h_{\widetilde{E}}^{\sigma(j) L}\left(x_{i}\right) \leqslant h_{\widetilde{F}}^{\sigma(j) L}\left(x_{i}\right) \leqslant h_{\widetilde{G}}^{\sigma(j) L}\left(x_{i}\right)$,
$h_{\widetilde{E}}^{\sigma(j) U}\left(x_{i}\right) \leqslant h_{\widetilde{F}}^{\sigma(j) U}\left(x_{i}\right) \leqslant h_{\widetilde{G}}^{\sigma(j) U}\left(x_{i}\right)$. Therefore, for any $p>0$, we have

$$
\begin{gathered}
\left|h_{\widetilde{E}}^{\sigma(j) L}\left(x_{i}\right)-h_{\widetilde{F}}^{\sigma(j) L}\left(x_{i}\right)\right|^{p} \leqslant\left|h_{\widetilde{E}}^{\sigma(j) L}\left(x_{i}\right)-h_{\widetilde{G}}^{\sigma(j) L}\left(x_{i}\right)\right|^{p}, \\
\left|h_{\widetilde{E}}^{\sigma(j) U}\left(x_{i}\right)-h_{\widetilde{F}}^{\sigma(j) U}\left(x_{i}\right)\right|^{p} \leqslant\left|h_{\widetilde{E}}^{\sigma(j) U}\left(x_{i}\right)-h_{\widetilde{G}}^{\sigma(j) U}\left(x_{i}\right)\right|^{p} .
\end{gathered}
$$

It follows that

$$
\begin{aligned}
& \left|h_{\widetilde{E}}^{\sigma(j) L}\left(x_{i}\right)-h_{\widetilde{F}}^{\sigma(j) L}\left(x_{i}\right)\right|^{p}+\left|h_{\widetilde{E}}^{\sigma(j) U}\left(x_{i}\right)-h_{\widetilde{F}}^{\sigma(j) U}\left(x_{i}\right)\right|^{p} \\
\leqslant & \left|h_{\widetilde{E}}^{\sigma(j) L}\left(x_{i}\right)-h_{\widetilde{G}}^{\sigma(j) L}\left(x_{i}\right)\right|^{p}+\left|h_{\widetilde{E}}^{\sigma(j) U}\left(x_{i}\right)-h_{\widetilde{G}}^{\sigma(j) U}\left(x_{i}\right)\right|^{p} .
\end{aligned}
$$

Hence,

$$
\begin{aligned}
& \frac{1}{2 n_{x_{i}}} \sum_{j=1}^{n_{x_{i}}}\left(\left|h_{\widetilde{E}}^{\sigma(j) L}\left(x_{i}\right)-h_{\widetilde{F}}^{\sigma(j) L}\left(x_{i}\right)\right|^{p}+\left|h_{\widetilde{E}}^{\sigma(j) U}\left(x_{i}\right)-h_{\widetilde{F}}^{\sigma(j) U}\left(x_{i}\right)\right|^{p}\right) \\
\leqslant & \frac{1}{2 n_{x_{i}}} \sum_{j=1}^{n_{x_{i}}}\left(\left|h_{\widetilde{E}}^{\sigma(j) L}\left(x_{i}\right)-h_{\widetilde{G}}^{\sigma(j) L}\left(x_{i}\right)\right|^{p}+\left|h_{\widetilde{E}}^{\sigma(j) U}\left(x_{i}\right)-h_{\widetilde{G}}^{\sigma(j) U}\left(x_{i}\right)\right|^{p}\right) .
\end{aligned}
$$

and

$$
\begin{aligned}
{\left[\frac{1}{2 n_{x_{i}}} \quad\right.} & \left.\sum_{j=1}^{n_{x_{i}}}\left(\left|h_{\widetilde{E}}^{\sigma(j) L}\left(x_{i}\right)-h_{\widetilde{F}}^{\sigma(j) L}\left(x_{i}\right)\right|^{p}+\left|h_{\widetilde{E}}^{\sigma(j) U}\left(x_{i}\right)-h_{\widetilde{F}}^{\sigma(j) U}\left(x_{i}\right)\right|^{p}\right)\right]^{\frac{1}{p}} \\
& \leqslant\left[\frac{1}{2 n_{x_{i}}} \sum_{j=1}^{n_{x_{i}}}\left(\left|h_{\widetilde{E}}^{\sigma(j) L}\left(x_{i}\right)-h_{\widetilde{G}}^{\sigma(j) L}\left(x_{i}\right)\right|^{p}+\left|h_{\widetilde{E}}^{\sigma(j) U}\left(x_{i}\right)-h_{\widetilde{G}}^{\sigma(j) U}\left(x_{i}\right)\right|^{p}\right)\right]^{\frac{1}{p}}
\end{aligned}
$$


Therefore,

$$
\begin{aligned}
& \frac{1}{n} \quad \sum_{i=1}^{n}\left[\frac{1}{2 n_{x_{i}}} \sum_{j=1}^{n_{x_{i}}}\left(\left|h_{\widetilde{E}}^{\sigma(j) L}\left(x_{i}\right)-h_{\widetilde{F}}^{\sigma(j) L}\left(x_{i}\right)\right|^{p}+\left|h_{\widetilde{E}}^{\sigma(j) U}\left(x_{i}\right)-h_{\widetilde{F}}^{\sigma(j) U}\left(x_{i}\right)\right|^{p}\right)\right]^{\frac{1}{p}} \\
& \leqslant \frac{1}{n} \sum_{i=1}^{n}\left[\frac{1}{2 n_{x_{i}}} \sum_{j=1}^{n_{x_{i}}}\left(\left|h_{\widetilde{E}}^{\sigma(j) L}\left(x_{i}\right)-h_{\widetilde{G}}^{\sigma(j) L}\left(x_{i}\right)\right|^{p}+\left|h_{\widetilde{E}}^{\sigma(j) U}\left(x_{i}\right)-h_{\widetilde{G}}^{\sigma(j) U}\left(x_{i}\right)\right|^{p}\right)\right]^{\frac{1}{p}} .
\end{aligned}
$$

That is, $d_{4}(\widetilde{E}, \widetilde{F}) \leqslant d_{4}(\widetilde{E}, \widetilde{G})$. Similarly, we can prove that $d_{4}(\widetilde{F}, \widetilde{G}) \leqslant d_{4}(\widetilde{E}, \widetilde{G})$. Thus $d_{4}(\widetilde{E}, \widetilde{F})$ satisfies the axiom (D5). The completes the proof.

Remark 2. $d_{5}(\widetilde{F}, \widetilde{G})$ is the special case of $d_{4}(\widetilde{F}, \widetilde{G})$ when $p=2 . d_{4}(\widetilde{F}, \widetilde{G})$ is called II-type generalized interval-valued hesitant fuzzy normalized distance measure and $d_{5}(\widetilde{F}, \widetilde{G})$ is called II-type interval- valued hesitant fuzzy normalized Euclidean distance measure corresponding to $d_{2}(\widetilde{F}, \widetilde{G})$. Accordingly, the interval-valued hesitant fuzzy normalized Hamming distance $d_{1}(\widetilde{F}, \widetilde{G})$ is a special case of II-type generalized interval-valued hesitant fuzzy normalized distance measures when $p=1$.

Based on Eq. (5), we can further define another measure $d_{4}^{\prime}(\widetilde{F}, \widetilde{G})$ between $\widetilde{F}$ and $\widetilde{G}$ as follows:

$$
d_{4}^{\prime}(\widetilde{F}, \widetilde{G})=\sum_{i=1}^{n}\left[\frac{1}{2 n_{x_{i}}} \sum_{j=1}^{n_{x_{i}}}\left(\left|h_{\widetilde{F}}^{\sigma(j) L}\left(x_{i}\right)-h_{\widetilde{G}}^{\sigma(j) L}\left(x_{i}\right)\right|^{p}+\left|h_{\widetilde{F}}^{\sigma(j) U}\left(x_{i}\right)-h_{\widetilde{G}}^{\sigma(j) U}\left(x_{i}\right)\right|^{p}\right)\right]^{\frac{1}{p}}, p>0 .
$$

Obviously, $d_{4}^{\prime}(\widetilde{F}, \widetilde{G})$ is not normalized and does not satisfy (D1). So it is not a distance measure as defined in Def. 4. (D1) is a rather restricted property and if it was modified, (D4) would need to be modified accordingly.

In the following, we will introduce another new distance measure which generalise (D1), i.e., the distance measure is defined in the $\mathbb{R}^{+}$, instead of $[0,1]$, in order to cover more general cases, accordingly, (D4) is also modified.

Definition 6. Let $\widetilde{F}$ and $\widetilde{G}$ be two IVHFSs on the reference set $X=\left\{x_{1}, x_{2}, \cdots, x_{n}\right\}$ and $d$ a real function $: \mathscr{I} \mathscr{V} \mathscr{H} \mathscr{F} \mathscr{S} \times \mathscr{I} \mathscr{V} \mathscr{H} \mathscr{F} \mathscr{S} \rightarrow \mathbb{R}^{*}$. $d$ is a distance measure between $\widetilde{F}$ and $\widetilde{G}$ if $d$ satisfies the following properties:
$\left(\mathrm{D} 1^{\prime}\right) 0 \leqslant d(\widetilde{F}, \widetilde{G})$;
$\left(\mathrm{D} 2^{\prime}\right) d(\widetilde{F}, \widetilde{G})=0 \Leftrightarrow \widetilde{F}=\widetilde{G}$;
$\left(\mathrm{D}^{\prime}\right) d(\widetilde{F}, \widetilde{G})=d(\widetilde{G}, \widetilde{F})$;
$\left(\mathrm{D} 4^{\prime}\right) d\left(\widetilde{F}, \widetilde{F}^{C}\right)=\max _{\widetilde{A}, \widetilde{B} \in \mathscr{I} \mathscr{V} \mathscr{H} \mathscr{F} \mathscr{S}(X)} d(\widetilde{A}, \widetilde{B})$ if and only if $\widetilde{F}=\{\langle x,\{[0,0]\}\rangle\}$ or $\widetilde{F}=\{\langle x,\{[1,1]\}\rangle\}$;

(D5') let $\widetilde{E}$ be an IVHFS and $\widetilde{E} \leqslant \widetilde{F} \leqslant \widetilde{G}$. Then
$d(\widetilde{E}, \widetilde{F}) \leqslant d(\widetilde{E}, \widetilde{G})$ and $d(\widetilde{F}, \widetilde{G}) \leqslant d(\widetilde{E}, \widetilde{G})$. where, $\left.\widetilde{F}^{C}=\left\{\left\langle x, \cup_{\widetilde{r} \in h_{\widetilde{F}(x)}}\left\{\widetilde{r}^{C}\right\}\right\}\right\rangle \mid x \in X\right\}$.

Corresponding to Def. $4, d(\widetilde{F}, \widetilde{G})$ in Definition 6 is called an interval-valued hesitant fuzzy distance between $\widetilde{F}$ and $\widetilde{G}$.

Remark 3. According to definitions, we can see that the normalized distance measure defined in Def. 4 is a special case of the distance measures defined in Def. 6. According to Def. 6, similar to the proof of Theorem 1, we have

Theorem 2. $d_{4}^{\prime}(\widetilde{F}, \widetilde{G})$ is an interval-valued hesitant fuzzy distance between $\widetilde{F}$ and $\widetilde{G}$.

Remark 4. The normalized distance measure can be obtained by a distance measure, i.e., if $d$ is a distance measure and $d_{\max }$ is the maximal distance of any two interval-valued hesitant fuzzy sets, then $\frac{d}{d_{\max }}$ is a normalized distance measure.

Remark 5. It is easy to prove that $\left(\mathrm{D} 5^{\prime}\right)$ is equiva- 
lent to

$\left(\mathrm{D}^{\prime \prime}\right)$ : Let $\widetilde{D}, \widetilde{E}, \widetilde{F}, \widetilde{G}$ be four IVHFSs and $\widetilde{D} \leqslant$ $\widetilde{E} \leqslant \widetilde{F} \leqslant \widetilde{G}$. Then $d(\widetilde{D}, \widetilde{G}) \geqslant d(\widetilde{E}, \widetilde{F})$.

\subsection{Interval-valued hesitant fuzzy $L_{p}$ distance based on $L_{p}$ metric}

In ${ }^{12}$, Huang studied the similarity of intuitionistic fuzzy sets based on $L_{p}$ metric. The reader can refer to ${ }^{12}$ about the $L_{p}$ metric concept. Huang extend the normal $L_{p}$ metric concept onto the one on the interval, this basic idea as follows:

Let $\Re$ be a Euclidean space and $I=\{[a, b] \mid a, b \in$ $\mathfrak{R}, a \leqslant b\}$ be the set of closed intervals in $\mathfrak{R}$. For any two interval $x=\left[x_{1}, y_{1}\right], y=\left[x_{2}, y_{2}\right] \in I$, the distance between two intervals $x, y$ is defined as follows:

$$
d_{p}(x, y)=\left(\left|x_{1}-x_{2}\right|^{p}+\left|y_{1}-y_{2}\right|^{p}\right)^{\frac{1}{p}}, p \geqslant 1 .
$$

This corresponds to representing an interval $[a, b] \in I$ as a point $(a, b)$, where the lower bounds of the in- tervals are represented in the $x$-axis, and the upper bounds in the $y$-axis, and then computing the $L_{p}$ distance between the points $\left(x_{1}, x_{2}\right)$ and $\left(y_{1}, y_{2}\right)$. Therefore, the distance $d_{p}$ is a suitable extension of the $L_{p}$ metric on the interval.

Hamming distance and Hausdorff distance are two important distance. Wei ${ }^{22}$ introduced a generalized interval-valued hesitant Hamming-Hausdorff distance which is a generalization of intervalvalued hesitant normal Hamming-Hausdorff distance. Since the IVHFEs of an IVHFS are sets of some intervals and the distance of two IVHFSs are closely related the distances of IVFHEs, we will introduce new distance measure named intervalvalued hesitant fuzzy $L_{p}$ distance whose limit is the interval-valued hesitant normal HammingHausdorff distance based on $L_{p}$ metric idea. This new measure between $\widetilde{F}$ and $\widetilde{G}$, denoted as $d_{p}(\widetilde{F}, \widetilde{G})$, is defined as follows:

$$
d_{p}(\widetilde{F}, \widetilde{G})=\frac{1}{n} \sum_{i=1}^{n}\left(\sum_{j=1}^{n_{x_{i}}}\left(\left|h_{\widetilde{F}}^{\sigma(j) L}\left(x_{i}\right)-h_{\widetilde{G}}^{\sigma(j) L}\left(x_{i}\right)\right|^{p}+\left|h_{\widetilde{F}}^{\sigma(j) U}\left(x_{i}\right)-h_{\widetilde{G}}^{\sigma(j) U}\left(x_{i}\right)\right|^{p}\right)\right)^{\frac{1}{p}}, p \geqslant 1 .
$$

It is easy to prove that $d_{p}(\widetilde{F}, \widetilde{G})$ is a distance measure in terms of Definition 3.

Wei ${ }^{21}$ introduced the interval-valued hesitant fuzzy normalized Hamming-Hausdorff distance as below:

$$
d_{7}(\widetilde{F}, \widetilde{G})=\frac{1}{n} \sum_{i=1}^{n}\left(\max _{j=1,2, \cdots, n_{x_{i}}}\left\{\left|h_{\widetilde{F}}^{\sigma(j) L}\left(x_{i}\right)-h_{\widetilde{G}}^{\sigma(j) L}\left(x_{i}\right)\right|,\left|h_{\widetilde{F}}^{\sigma(j) U}\left(x_{i}\right)-h_{\widetilde{G}}^{\sigma(j) U}\left(x_{i}\right)\right|\right\}\right)
$$

In the following, we propose and prove an interesting result:

$$
\lim _{p \rightarrow+\infty} d_{p}(\widetilde{F}, \widetilde{G})=d_{7}(\widetilde{F}, \widetilde{G}) .
$$

To prove the interesting result Eq. (9), the following lemma is necessary.

Lemma $1 .{ }^{12}$ Let $a, b$ be two non-negative real numbers. We have

$$
\lim _{p \rightarrow+\infty}\left(a^{p}+b^{p}\right)^{\frac{1}{p}}=\max \{a, b\}, p \geqslant 1 .
$$

The following Theorem 3 can be easily obtained from Lemma 1.

Theorem 3. $\lim _{p \rightarrow+\infty} d_{p}(\widetilde{F}, \widetilde{G})=d_{7}(\widetilde{F}, \widetilde{G})$.

Proof. By the properties of a limit and Lemma 1, we have 


$$
\begin{aligned}
\lim _{p \rightarrow+\infty} d_{p}(\widetilde{F}, \widetilde{G}) & =\lim _{p \rightarrow+\infty}\left(\frac{1}{n} \sum_{i=1}^{n}\left(\sum_{j=1}^{n_{x_{i}}}\left(\left|h_{\widetilde{F}}^{\sigma(j) L}\left(x_{i}\right)-h_{\widetilde{G}}^{\sigma(j) L}\left(x_{i}\right)\right|^{p}+\left|h_{\widetilde{F}}^{\sigma(j) U}\left(x_{i}\right)-h_{\widetilde{G}}^{\sigma(j) U}\left(x_{i}\right)\right|^{p}\right)\right)^{\frac{1}{p}}\right) \\
& =\frac{1}{n} \sum_{i=1}^{n} \lim _{p \rightarrow+\infty}\left(\sum_{j=1}^{n_{x_{i}}}\left(\left|h_{\widetilde{F}}^{\sigma(j) L}\left(x_{i}\right)-h_{\widetilde{G}}^{\sigma(j) L}\left(x_{i}\right)\right|^{p}+\left|h_{\widetilde{F}}^{\sigma(j) U}\left(x_{i}\right)-h_{\widetilde{G}}^{\sigma(j) U}\left(x_{i}\right)\right|^{p}\right)\right)^{\frac{1}{p}} \\
& =\frac{1}{n} \sum_{i=1}^{n}\left(\max _{j=1,2, \cdots, n_{x_{i}}}\left\{\left|h_{\widetilde{F}}^{\sigma(j) L}\left(x_{i}\right)-h_{\widetilde{G}}^{\sigma(j) L}\left(x_{i}\right)\right|,\left|h_{\widetilde{F}}^{\sigma(j) U}\left(x_{i}\right)-h_{\widetilde{G}}^{\sigma(j) U}\left(x_{i}\right)\right|\right\}\right) \\
& =d_{7}(\widetilde{F}, \widetilde{G}) .
\end{aligned}
$$

In the multiple attribute decision making problems, when we select different distance functions, it may lead to different results. Usually, the weight of each element $x_{i} \in X$ should be taken into account. Hereafter, assume that the weight of the element $x_{i} \in X$ is $\omega_{i}(i=1,2, \cdots, n)$ with $\omega_{i} \in[0,1]$ and $\sum_{i=1}^{n} \omega_{i}=1$. Several weighted distance measures for the IVHFSs have been presented in ${ }^{21}$. Among all weighted distance measures for the IVHFSs, the most popular one is the generalized interval-valued hesitant fuzzy weighted Hamming distance. For two IVHFSs $\widetilde{F}$ and $\widetilde{G}$, it is defined as below:

$$
d_{8}(\widetilde{F}, \widetilde{G})=\left[\sum_{i=1}^{n} \omega_{i}\left(\frac{1}{2 n_{x_{i}}} \sum_{j=1}^{n_{x_{i}}}\left(\left|h_{\widetilde{F}}^{\sigma(j) L}\left(x_{i}\right)-h_{\widetilde{G}}^{\sigma(j) L}\left(x_{i}\right)\right|^{p}+\left|h_{\widetilde{F})}^{\sigma(j) U}\left(x_{i}\right)-h_{\widetilde{G}}^{\sigma(j) U}\left(x_{i}\right)\right|^{p}\right)\right]^{\frac{1}{p}}, p>0\right.
$$

Here, we define a generalized interval-valued hesitant fuzzy weighted $L_{p}$ distance as below:

$$
d_{9}(\widetilde{F}, \widetilde{G})=\sum_{i=1}^{n} \omega_{i}\left(\sum_{j=1}^{n_{x_{i}}}\left(\left|h_{\widetilde{F}}^{\sigma(j) L}\left(x_{i}\right)-h_{\widetilde{G}}^{\sigma(j) L}\left(x_{i}\right)\right|^{p}+\left|h_{\widetilde{F}}^{\sigma(j) U}\left(x_{i}\right)-h_{\widetilde{G}}^{\sigma(j) U}\left(x_{i}\right)\right|^{p}\right)\right)^{\frac{1}{p}}, p \geqslant 1 .
$$

It is also easy to prove that $d_{9}(\widetilde{F}, \widetilde{G})$ is a distance measure in terms of Definition 6.

Obviously, if each element has the same importance, i.e., $\omega_{i}=\frac{1}{n}$, then Eq. (10) and Eq. (11) will be degenerated to Eq. (3) and Eq. (7) respectively.

\section{New Similarity Measures for IVHFSs}

In this section, we focus on the new similarity measures in IVHFSs. The existing similarity measures of IVHFSs are analysed and then a new kind of new and more general similarity of IVHFSs are given; whist a new similarity measure, which is not dependent on the distance measure, but based on the settheoretic approach, is also introduced in this section.

\subsection{Analysis on the existing similarity measures for IVHFSs}

Similarity measures are generally used for determining the degree of similarity between two objects. Similarity measure between two fuzzy sets is mostly dependent on their corresponding distance measures, so the former can normally be derived from the latter. This is also true for IVHFSs as detailed in ${ }^{21}$. In addition, in most of cases, the relation between the similarity measure $s$ and the distance measure $d$ defined in the interval $[0,1]$ for IVHFSs can be naturally expressed as:

$$
s=1-d .
$$


However, there are also some other ways or formulas to derive the similarity measure from the distance measure for IVHFSs. For example, a new similarity measure is defined with the following theorem.

Theorem $4^{7}$ Let $\widetilde{F}$ and $\widetilde{G}$ be two IVHFSs, $Z$ : $[0,1] \rightarrow[0,1]$ a strictly monotone decreasing real function, and $d$ a distance measure between $\widetilde{F}$ and $\widetilde{G}$. Then,

$$
s_{d}(\widetilde{F}, \widetilde{G})=\frac{Z(d(\widetilde{F}, \widetilde{G}))-Z(1)}{Z(0)-Z(1)}
$$

is a similarity measure between $\widetilde{F}$ and $\widetilde{G}$ based on the corresponding distance $d$.

As indicated in ${ }^{7}$, the distance $d$ is a normalized distance in terms if Definition 4 , so $0 \leqslant d(\widetilde{F}, \widetilde{G}) \leqslant 1$. In Section 3, we have the modified normalized distance with the update of the axiom $(\mathrm{D}(1))$ : $0 \leqslant d(\widetilde{F}, \widetilde{G}) \leqslant 1$ to $\left(\mathrm{D} 1^{\prime}\right): 0 \leqslant d(\widetilde{F}, \widetilde{G}) \leqslant d_{\max }$, where $d_{\text {max }} \in \mathbb{R}^{+}$is the maximal distance. Hence we can give an extension of Theorem 4 .

Theorem 5. Let $\widetilde{F}$ and $\widetilde{G}$ be two IVHFSs, $Z$ : $[0,1] \rightarrow[0,1]$ a strictly monotone decreasing real function, $d$ a distance measure between $\widetilde{F}$ and $\widetilde{G}$, and $d_{\max }$ the maximal distance. Then,

$$
s_{d}^{\prime}(\widetilde{F}, \widetilde{G})=\frac{Z(d(\widetilde{F}, \widetilde{G}))-Z\left(d_{\max }\right)}{Z(0)-Z\left(d_{\max }\right)}
$$

is a similarity measure between $\widetilde{F}$ and $\widetilde{G}$ based on the corresponding distance $d$.

Proof. It only needs to prove that the measure $s_{d}^{\prime}(\widetilde{F}, \widetilde{G})$ is a similarity measure in terms of Definition 7. Since $Z$ is a monotone decreasing function and $0 \leqslant d(\widetilde{F}, \widetilde{G}) \leqslant d_{\max }$, we have

$$
\left.Z\left(d_{\max }\right)\right) \leqslant Z(d(\widetilde{F}, \widetilde{G}) \leqslant Z(0) .
$$

It follows that

$$
Z(d(\widetilde{F}, \widetilde{G}))-Z\left(d_{\max }\right) \leqslant Z(0)-Z\left(d_{\max }\right) .
$$

Therefore,

$$
0 \leqslant \frac{Z(d(\widetilde{F}, \widetilde{G}))-Z\left(d_{\max }\right)}{Z(0)-Z\left(d_{\max }\right)} \leqslant 1 .
$$

Hence (S1) holds. Similarly, we can prove (S5) holds according to (D5) in Definition 6, the details are omitted. For (S2)-(S4) are straightforward.

By Theorem 4 and Theorem 5, if we choose the monotone decreasing function $Z(x)=e^{-x}$, or $1-x$, or $\frac{1-x}{1+x}$, or others), then different similarity measures between $\widetilde{F}$ and $\widetilde{G}$ will be obtained, and they are closely related to the distance. It is also consistent with most similarity measures corresponding to distant. Hence, Theorem 4 and Theorem 5 present two ways to construct the similarity measures for IVHFSs based on some distance measures. For example, if we choose the function $Z(x)=1-x$ in Theorem 5, then we have $s_{d}^{\prime}(\widetilde{F}, \widetilde{G})=1-\frac{d(\widetilde{F}, \widetilde{G})}{d_{\max }}$. If the distance $d$ is normalized into [0,1], then we have $s_{d}(\widetilde{F}, \widetilde{G})=1-d(\widetilde{F}, \widetilde{G})$, which is consistent with Eq. (12). This means both $s_{d}$ and $s_{d}^{\prime}$ are more general definitions of similarity measures than the most common case as in Eq. (12). Based on this idea and different distance measures for IVHFSs defined in Section 2, we can construct the following new similarity measures accordingly:

- Similarity measure for IVHFSs based on IItype generalized interval-valued hesitant fuzzy distance measures:

$$
s_{1}(\widetilde{F}, \widetilde{G})=1-\frac{1}{n} \sum_{i=1}^{n}\left[\frac{1}{2 n_{x_{i}}} \sum_{j=1}^{n_{x_{i}}}\left(\left|h_{\widetilde{F}}^{\sigma(j) L}\left(x_{i}\right)-h_{\widetilde{G}}^{\sigma(j) L}\left(x_{i}\right)\right|^{p}+\left|h_{\widetilde{F}}^{\sigma(j) U}\left(x_{i}\right)-h_{\widetilde{G}}^{\sigma(j) U}\left(x_{i}\right)\right|^{p}\right)\right]^{\frac{1}{p}}, p>0
$$


If we take the weight of each element $x \in X$ into account in $s_{1}(\widetilde{F}, \widetilde{G})$ and $s_{2}(\widetilde{F}, \widetilde{G})$, where $\omega=$ $\left(\omega_{1}, \omega_{2}, \cdots, \omega_{n}\right), \omega_{i} \in[0,1]$ and $\sum_{i=1}^{n} \omega_{i}=1$. Then we obtain
- Similarity measure with weight $\omega$ for IVHFSs based on II-type generalized interval-valued hesitant fuzzy distance measures

$$
s_{1}^{\prime}(\widetilde{F}, \widetilde{G})=1-\sum_{i=1}^{n} \omega_{i}\left[\frac{1}{2 n_{x_{i}}} \sum_{j=1}^{n_{x_{i}}}\left(\left|h_{\widetilde{F}}^{\sigma(j) L}\left(x_{i}\right)-h_{\widetilde{G}}^{\sigma(j) L}\left(x_{i}\right)\right|^{p}+\left|h_{\widetilde{F}}^{\sigma(j) U}\left(x_{i}\right)-h_{\widetilde{G}}^{\sigma(j) U}\left(x_{i}\right)\right|^{p}\right)\right]^{\frac{1}{p}}, p>0
$$

- Similarity measure for IVHFSs based on interval-valued hesitant fuzzy $L_{p}$ distance

$$
s_{2}(\widetilde{F}, \widetilde{G})=1-\frac{1}{n} \sum_{i=1}^{n}\left(\sum_{j=1}^{n_{x_{i}}}\left(\left|h_{\widetilde{F}}^{\sigma(j) L}\left(x_{i}\right)-h_{\widetilde{G}}^{\sigma(j) L}\left(x_{i}\right)\right|^{p}+\left|h_{\widetilde{F}}^{\sigma(j) U}\left(x_{i}\right)-h_{\widetilde{G}}^{\sigma(j) U}\left(x_{i}\right)\right|^{p}\right)\right)^{\frac{1}{p}}, p \geqslant 1 .
$$

- Similarity measure with weight $\omega$ for IVHFSs

$$
s_{2}^{\prime}(\widetilde{F}, \widetilde{G})=1-\sum_{i=1}^{n} \omega_{i}\left(\sum_{j=1}^{n_{x_{i}}}\left(\left|h_{\widetilde{F}}^{\sigma(j) L}\left(x_{i}\right)-h_{\widetilde{G}}^{\sigma(j) L}\left(x_{i}\right)\right|^{p}+\left|h_{\widetilde{F}}^{\sigma(j) U}\left(x_{i}\right)-h_{\widetilde{G}}^{\sigma(j) U}\left(x_{i}\right)\right|^{p}\right)\right)^{\frac{1}{p}}, p \geqslant 1 .
$$

Of course, we can also choose other types of monotone decreasing functions to construct other different similarity measures for IVHFSs. Many similarity measures can be obtained by the relation function: $s_{d}(\widetilde{F}, \widetilde{G})=1-d(\widetilde{F}, \widetilde{G})$ or $s_{d}^{\prime}(\widetilde{F}, \widetilde{G})=$ $1-\frac{d(\widetilde{F}, \widetilde{G})}{d_{\max }}$, will not be listed one by one here.

\subsection{New similarity measures for IVHFSs based on set-theoretic approach}

It follows from the above analysis on the existing similarity measures that most of similarity measures are dependent on the corresponding distance measures. In this section, we will introduce a new similarity measure for IVHFSs, which is not dependent on the distance measure, but based on the set-theoretic approach.

Theorem 6. Let $\widetilde{F}, \widetilde{G}$ be two IVHFSs. Then

$$
s_{3}(\widetilde{F}, \widetilde{G})=\frac{1}{n} \sum_{i=1}^{n} \min \left\{\frac{\sum_{j=1}^{n_{x_{i}}} \min \left\{h_{\widetilde{F}}^{\sigma(j) L}\left(x_{i}\right), h_{\widetilde{G}}^{\sigma(j) L}\left(x_{i}\right)\right\}}{\sum_{j=1}^{n_{x_{i}}} \max \left\{h_{\widetilde{F}}^{\sigma(j) L}\left(x_{i}\right), h_{\widetilde{G}}^{\sigma(j) L}\left(x_{i}\right)\right\}}, \frac{\sum_{j=1}^{n_{x_{i}}} \min \left\{\left(h_{\widetilde{F}}^{\sigma(j) U}\left(x_{i}\right)\right)^{c},\left(h_{\widetilde{G}}^{\sigma(j) U}\left(x_{i}\right)\right)^{c}\right\}}{\sum_{j=1}^{n_{x_{i}}} \max \left\{\left(h_{\widetilde{F}}^{\sigma(j) U}\left(x_{i}\right)\right)^{c},\left(h_{\widetilde{G}}^{\sigma(j) U}\left(x_{i}\right)\right)^{c}\right\}}\right\}
$$

is a similarity measure between $\widetilde{F}$ and $\widetilde{G}$.

Proof. It needs to prove that $s_{3}(\widetilde{F}, \widetilde{G})$ satisfies (S1)-(S5) in Definition 5. Obviously, $s_{3}(\widetilde{F}, \widetilde{G})$ sat- isfies (S1), (S3) and (S4). Now, we need to prove $s_{3}(\widetilde{F}, \widetilde{G})$ satisfies (S2) and (S5).

For $(\mathrm{S} 2), s_{3}(\widetilde{F}, \widetilde{G})=1$, that is, 


$$
\frac{1}{n} \sum_{i=1}^{n} \min \left\{\frac{\sum_{j=1}^{n_{x_{i}}} \min \left\{h_{\widetilde{F}}^{\sigma(j) L}\left(x_{i}\right), h_{\widetilde{G}}^{\sigma(j) L}\left(x_{i}\right)\right\}}{\sum_{j=1}^{n_{x_{i}}} \max \left\{h_{\widetilde{F}}^{\sigma(j) L}\left(x_{i}\right), h_{\widetilde{G}}^{\sigma(j) L}\left(x_{i}\right)\right\}}, \frac{\sum_{j=1}^{n_{x_{i}}} \min \left\{\left(h_{\widetilde{F}}^{\sigma(j) U}\left(x_{i}\right)\right)^{c},\left(h_{\widetilde{G}}^{\sigma(j) U}\left(x_{i}\right)\right)^{c}\right\}}{\sum_{j=1}^{n_{x_{i}}} \max \left\{\left(h_{\widetilde{F}}^{\sigma(j) U}\left(x_{i}\right)\right)^{c},\left(h_{\widetilde{G}}^{\sigma(j) U}\left(x_{i}\right)\right)^{c}\right\}}\right\}=1
$$

if and only if

$$
\begin{aligned}
& \min \left\{h_{\widetilde{F}}^{\sigma(j) L}\left(x_{i}\right), h_{\widetilde{G}}^{\sigma(j) L}\left(x_{i}\right)\right\} \\
= & \max \left\{h_{\widetilde{F}}^{\sigma(j) L}\left(x_{i}\right), h_{\widetilde{G}}^{\sigma(j) L}\left(x_{i}\right)\right\}
\end{aligned}
$$

and

$$
\begin{aligned}
& \min \left\{\left(h_{\widetilde{F}}^{\sigma(j) U}\left(x_{i}\right)\right)^{c},\left(h_{\widetilde{G}}^{\sigma(j) U}\left(x_{i}\right)\right)^{c}\right\} \\
= & \max \left\{\left(h_{\widetilde{F}}^{\sigma(j) U}\left(x_{i}\right)\right)^{c},\left(h_{\widetilde{G}}^{\sigma(j) U}\left(x_{i}\right)\right)^{c}\right\}
\end{aligned}
$$

if and only if

$$
h_{\widetilde{F}}^{\sigma(j) L}\left(x_{i}\right)=h_{\widetilde{G}}^{\sigma(j) L}\left(x_{i}\right) \text { and } h_{\widetilde{F}}^{\sigma(j) U}\left(x_{i}\right)=h_{\widetilde{G}}^{\sigma(j) U}\left(x_{i}\right)
$$

if and only if $\widetilde{F}=\widetilde{G}$.

For (S5). Let $\widetilde{E}, \widetilde{F}$, and $\widetilde{G}$ be three IVHFSs and $\widetilde{E} \leqslant \widetilde{F} \leqslant \widetilde{G}$.

$$
\begin{aligned}
s_{3}(\widetilde{E}, \widetilde{F}) & =\frac{1}{n} \sum_{i=1}^{n} \min \left\{\frac{\sum_{j=1}^{n_{x_{i}}} \min \left\{h_{\widetilde{E}}^{\sigma(j) L}\left(x_{i}\right), h_{\widetilde{F}}^{\sigma(j) L}\left(x_{i}\right)\right\}}{\sum_{j=1}^{n_{x_{i}}} \max \left\{h_{\widetilde{E}}^{\sigma(j) L}\left(x_{i}\right), h_{\widetilde{F}}^{\sigma(j) L}\left(x_{i}\right)\right\}}, \frac{\sum_{j=1}^{n_{x_{i}}} \min \left\{\left(h_{\widetilde{E}}^{\sigma(j) U}\left(x_{i}\right)\right)^{c},\left(h_{\widetilde{F}}^{\sigma(j) U}\left(x_{i}\right)\right)^{c}\right\}}{\sum_{j=1}^{n_{x_{i}}} \max \left\{\left(h_{\widetilde{E}}^{\sigma(j) U}\left(x_{i}\right)\right)^{c},\left(h_{\widetilde{F}}^{\sigma(j) U}\left(x_{i}\right)\right)^{c}\right\}}\right\} \\
& =\frac{1}{n} \sum_{i=1}^{n} \min \left\{\frac{\sum_{j=1}^{n_{x_{i}}} h_{\widetilde{E}}^{\sigma(j) L}\left(x_{i}\right)}{\sum_{j=1}^{n_{x_{i}}} h_{\widetilde{E}}^{\sigma(j) L}\left(x_{i}\right)}, \frac{\sum_{j=1}^{n_{x_{i}}}\left(h_{\widetilde{F}}^{\sigma(j) U}\left(x_{i}\right)\right)^{c}}{\sum_{j=1}^{n_{x_{i}}}\left(h_{\widetilde{F}}^{\sigma(j) U}\left(x_{i}\right)\right)^{c}}\right\} \\
& \geqslant \frac{1}{n} \sum_{i=1}^{n} \min \left\{\frac{\sum_{j=1}^{n_{x_{i}}} h_{\widetilde{E}}^{\sigma(j) L}\left(x_{i}\right)}{\sum_{j=1}^{n_{x_{i}}} h_{\widetilde{E}}^{\sigma(j) L}\left(x_{i}\right)}, \frac{\sum_{j=1}^{n_{x_{i}}}\left(h_{\widetilde{G}}^{\sigma(j) U}\left(x_{i}\right)\right)^{c}}{\sum_{j=1}^{n_{x_{i}}}\left(h_{\widetilde{G}}^{\sigma(j) U}\left(x_{i}\right)\right)^{c}}\right\} \\
& =\frac{1}{n} \sum_{i=1}^{n} \min \left\{\frac{\sum_{j=1}^{n_{x_{i}}} \min \left\{h_{\widetilde{E}}^{\sigma(j) L}\left(x_{i}\right), h_{\widetilde{G}}^{\sigma(j) L}\left(x_{i}\right)\right\}}{\sum_{j=1}^{n_{x_{i}}} \max \left\{h_{\widetilde{E}}^{\sigma(j) L}\left(x_{i}\right), h_{\widetilde{G}}^{\sigma(j) L}\left(x_{i}\right)\right\}}, \frac{\sum_{j=1}^{n_{x_{i}}} \min \left\{\left(h_{\widetilde{E}}^{\sigma(j) U}\left(x_{i}\right)\right)^{c},\left(h_{\widetilde{G}}^{\sigma(j) U}\left(x_{i}\right)\right)^{c}\right\}}{\sum_{j=1}^{n_{x_{i}}} \max \left\{\left(h_{\widetilde{E}}^{\sigma(j) U}\left(x_{i}\right)\right)^{c},\left(h_{\widetilde{G}}^{\sigma(j) U}\left(x_{i}\right)\right)^{c}\right\}}\right\} \\
& =s_{3}(\widetilde{E}, \widetilde{G}) .
\end{aligned}
$$

Similarly, we have $s_{3}(\widetilde{E}, \widetilde{G}) \leqslant s_{3}(\widetilde{F}, \widetilde{G})$. Therefore, $s_{3}$ is a similarity measure for IVHFSs according to Definition 5.
In fact, we can also propose another new measure between $\widetilde{F}$ and $\widetilde{G}$ as follows:

$$
s_{4}(\widetilde{F}, \widetilde{G})=\frac{1}{n} \sum_{i=1}^{n} \min \left\{\frac{\sum_{j=1}^{n_{x_{i}}} \min \left\{h_{\widetilde{F}}^{\sigma(j) L}\left(x_{i}\right), h_{\widetilde{G}}^{\sigma(j) U}\left(x_{i}\right)\right\}}{\sum_{j=1}^{n_{x_{i}}} \max \left\{h_{\widetilde{F}}^{\sigma(j) L}\left(x_{i}\right), h_{\widetilde{G}}^{\sigma(j) U}\left(x_{i}\right)\right\}}, \frac{\sum_{j=1}^{n_{x_{i}}} \min \left\{\left(h_{\widetilde{F}}^{\sigma(j) U}\left(x_{i}\right)\right)^{c},\left(h_{\widetilde{G}}^{\sigma(j) L}\left(x_{i}\right)\right)^{c}\right\}}{\sum_{j=1}^{n_{x_{i}}} \max \left\{\left(h_{\widetilde{F}}^{\sigma(j) U}\left(x_{i}\right)\right)^{c},\left(h_{\widetilde{G}}^{\sigma(j) L}\left(x_{i}\right)\right)^{c}\right\}}\right\} .
$$

Similar to the proof of Theorem 6, we have

Theorem 7. $s_{4}(\widetilde{F}, \widetilde{G})$ is a similarity measure between $\widetilde{F}$ and $\widetilde{G}$.
By comparing $s_{3}(\widetilde{F}, \widetilde{G})$ and $s_{4}(\widetilde{F}, \widetilde{G})$, we can find that they have a dual relationship. In this sense, a compromise between $s_{3}(\widetilde{F}, \widetilde{G})$ and $s_{4}(\widetilde{F}, \widetilde{G})$ is 
meaningful so that we can have a new measure as a weighted average of $s_{3}(\widetilde{F}, \widetilde{G})$ and $s_{4}(\widetilde{F}, \widetilde{G})$ as follows:

$s_{5}(\widetilde{F}, \widetilde{G})=\omega s_{3}(\widetilde{F}, \widetilde{G})+(1-\omega) s_{4}(\widetilde{F}, \widetilde{G}), 0 \leqslant \omega \leqslant 1$.

It follows from Theorem 6 and Theorem 7 that
Theorem 8. $s_{5}(\widetilde{F}, \widetilde{G})$ is a similarity measure between $\widetilde{F}$ and $\widetilde{G}$.

The proof of Theorem 8 is easy from Theorem 7 and Theorem 6 , so we omit its proof.

If we take the weight of each element $x \in X$ into account in $s_{3}(\widetilde{F}, \widetilde{G})$ and $s_{4}(\widetilde{F}, \widetilde{G})$, then we obtain

$$
s_{3}^{\prime}(\widetilde{F}, \widetilde{G})=\sum_{i=1}^{n} \omega_{i}\left[\min \left\{\frac{\sum_{j=1}^{n_{x_{i}}} \min \left\{h_{\widetilde{F}}^{\sigma(j) L}\left(x_{i}\right), h_{\widetilde{G}}^{\sigma(j) L}\left(x_{i}\right)\right\}}{\sum_{j=1}^{n_{x_{i}}} \max \left\{h_{\widetilde{F}}^{\sigma(j) L}\left(x_{i}\right), h_{\widetilde{G}}^{\sigma(j) L}\left(x_{i}\right)\right\}}, \frac{\sum_{j=1}^{n_{x_{i}}} \min \left\{\left(h_{\widetilde{F}}^{\sigma(j) U}\left(x_{i}\right)\right)^{c},\left(h_{\widetilde{G}}^{\sigma(j) U}\left(x_{i}\right)\right)^{c}\right\}}{\sum_{j=1}^{n_{x_{i}}} \max \left\{\left(h_{\widetilde{F}}^{\sigma(j) U}\left(x_{i}\right)\right)^{c},\left(h_{\widetilde{G}}^{\sigma(j) U}\left(x_{i}\right)\right)^{c}\right\}}\right\}\right]
$$

and

$$
s_{4}^{\prime}(\widetilde{F}, \widetilde{G})=\sum_{i=1}^{n} \omega_{i}\left[\min \left\{\frac{\sum_{j=1}^{n_{x_{i}}} \min \left\{h_{\widetilde{F}}^{\sigma(j) L}\left(x_{i}\right), h_{\widetilde{G}}^{\sigma(j) U}\left(x_{i}\right)\right\}}{\sum_{j=1}^{n_{x_{i}}} \max \left\{h_{\widetilde{F}}^{\sigma(j) L}\left(x_{i}\right), h_{\widetilde{G}}^{\sigma(j) U}\left(x_{i}\right)\right\}}, \frac{\sum_{j=1}^{n_{x_{i}}} \min \left\{\left(h_{\widetilde{F}}^{\sigma(j) U}\left(x_{i}\right)\right)^{c},\left(h_{\widetilde{G}}^{\sigma(j) L}\left(x_{i}\right)\right)^{c}\right\}}{\sum_{j=1}^{n_{x_{i}}} \max \left\{\left(h_{\widetilde{F}}^{\sigma(j) U}\left(x_{i}\right)\right)^{c},\left(h_{\widetilde{G}}^{\sigma(j) L}\left(x_{i}\right)\right)^{c}\right\}}\right\}\right],
$$

where $\omega_{i} \in[0,1]$ and $\sum_{i=1}^{n} \omega_{i}=1$. Specially, if $\omega_{i}=\frac{1}{n}(i=1,2, \cdots, n)$, then Eq. (20) and Eq. (21) will be degenerated to Eq. (17) and Eq. (18), respectively.

\section{A Multiple Attribute Decision Making Approach Based on Interval-Valued Hesitant Fuzzy Information}

In this section, we shall utilize the interval-valued hesitant fuzzy similarity measures to multiple attribute decision making with interval-valued hesitant fuzzy information, especially, a relative similarity measure is proposed in order to fit into the multiple attribute decision making approach.

Assume $A=\left\{A_{1}, A_{2}, \cdots, A_{m}\right\}$ is a discrete set of alternatives, $X=\left\{x_{1}, x_{2}, \cdots, x_{n}\right\}$ is a set of attributes, and $\omega=\left\{\omega_{1}, \omega_{2}, \cdots, \omega_{n}\right\}$ is the weight vector of the attribute $x_{j}(j=1,2, \cdots, n)$, where $\omega_{j} \in[0,1]$ and $\sum_{j=1}^{n}=1$. Assume that the characteristics for alternatives $A_{i}$ are presented by the IVHFS as follows:

$$
A_{i}=\left\{\left\langle x_{j}, h_{A_{i}}\left(x_{j}\right)\right\rangle \mid x_{j} \in X\right\}, i=1,2, \cdots, m,
$$

where $h_{A_{i}}\left(x_{j}\right)$ indicates the degree that the alterna- tive $A_{i}$ satisfies the attribute $x_{j}$. This also means that, if the decision makers provide several intervalvalued values for the alternative $A_{i}$ under the attribute $x_{j}$ on condition of anonymity, these values can be considered as an interval-valued hesitant fuzzy element $h_{A_{i}}\left(x_{j}\right)$. Before the decision making method is introduced, we firstly introduce the concepts of the positive ideal IVHFS, the negative ideal IVHFS and the relative similarity measure for IVHFS as follows:

Definition 7. ${ }^{14}$ Let $A_{i}(i=1,2, \cdots, m)$ be IVHFSs on the reference set $X$. The positive ideal IVHFS $A^{+}$ and the negative ideal IVHFS $A^{-}$are defined as follows, respectively:

$$
\begin{aligned}
& A^{+}=\left\{\left\langle x_{j}, h_{A^{+}}\left(x_{j}\right)\right\rangle \mid x_{j} \in X\right\}, \\
& A^{-}=\left\{\left\langle x_{j}, h_{A^{-}}\left(x_{j}\right)\right\rangle \mid x_{j} \in X\right\},
\end{aligned}
$$

where,

$$
\begin{aligned}
& h_{A^{+}}\left(x_{j}\right)=\left\{\max _{i}\left\{h_{A_{i}}^{\sigma(k)}\left(x_{j}\right)\right\} \mid k=1,2, \cdots, n_{x_{i}},\right. \\
& i=1,2, \cdots, m\} \\
& h_{A^{-}}\left(x_{j}\right)=\left\{\min _{i}\left\{h_{A_{i}}^{\sigma(k)}\left(x_{j}\right)\right\} \mid k=1,2, \cdots, n_{x_{i}},\right. \\
& i=1,2, \cdots, m\} .
\end{aligned}
$$


Note that both the positive ideal IVHFS and the negative ideal IVHFS are special case of an IVHFS with only one IVHFE in each IVHFS.

In order to choose the desired alternative in multiple attribute decision making problem, based on some aforementioned similarity measures for IVHFSs in Section 4, we can compute the similarity degree between the positive ideal IVHFS $A^{+}$and the alternative $A_{i}$, as well as the similarity degree between the negative ideal IVHFS $A^{-}$ and the alternative $A_{i}$, denoted by $s\left(A^{+}, A_{i}\right)$ and $s\left(A^{-}, A_{i}\right)$, respectively. Intuitively, the larger the similarity $s\left(A^{+}, A_{i}\right)$, the better the alternative; while the smaller the $s\left(A^{-}, A_{i}\right)$, the better the alternative. Motivated by the well-known TOPSIS (Technique for Order Preference by Similarity to an Ideal Solution method ${ }^{4}$, we take both $s\left(A^{+}, A_{i}\right)$ and $s\left(A^{-}, A_{i}\right)$ into consideration. This leads naturally to the concept of relative similarity measure.

Definition 8. Let $A=\left\{A_{1}, A_{2}, \cdots, A_{m}\right\}$ be a discrete set of alternatives. The relative similarity measures $r s_{i}$ corresponding to the alternative $A_{i}$ are defined as:

$$
r s_{i}=\frac{s\left(A^{+}, A_{i}\right)}{s\left(A^{+}, A_{i}\right)+s\left(A^{-}, A_{i}\right)}, i=1,2, \cdots, m \text {. }
$$

Based on the above analysis and concepts, we propose the following procedure of a multiple attribute decision making:

Step 1. Construct the corresponding intervalvalued hesitant fuzzy sets according to the characteristics of the alternatives:

$$
A_{i}=\left\{\left\langle x_{j}, h_{A_{i}}\left(x_{j}\right)\right\rangle \mid x_{j} \in X\right\}, i=1,2, \cdots, m .
$$

Step 2. Construct the notions of the positive ideal IVHFS $A^{+}$and the negative ideal IVHFS $A^{-}$ according to Def.7.
Step 3. Calculate similarity measure $s\left(A^{+}, A_{i}\right)$ and $s\left(A^{-}, A_{i}\right)$ according to some similarity measures given in the Section 4 . We will choose any weighted similarity measures when the attributes with weight information. Otherwise, we can choose other similarity measures without weight.

Step 4. Calculate the relative similarity measure $r s_{i}$ corresponding to the alternative $A_{i}$ according to Eq.(23).

Step 5. Obtain the priority of the alternative $A_{i}(i=1,2, \cdots, m)$ by ranking the $r s_{i}(i=$ $1,2, \cdots, m)$.

Step 6. End of the steps.

\section{An Example Illustration}

We choose a practical problem as an illustrative example, which have been discussed in ${ }^{22}$, in order to illustrate the effeteness of the proposed method compared with the the one in ${ }^{22}$.

Assume that there is an investment company, which wants to invest a sum of money in the best option. There is a panel with five possible alternatives $A_{i}(i=1,2, \cdots, 5)$ to invest money: (1) $A_{1}$ is a car company; (2) $A_{2}$ is a food company; (3) $A_{3}$ is a computer company; (4) $A_{4}$ is an cosmetic company; (5) $A_{5}$ is a TV company. The investment company must take a decision according to the following attributes $x_{i}(i=1,2,3,4)$ : (1) $x_{1}$ is the risk analysis; (2) $x_{2}$ is the growth analysis; (3) $x_{3}$ is the social-political impact analysis; (4) $x_{4}$ is the environment impact analysis. In order to avoid influence each other, the decision makers are required to evaluate the five companies $A_{i}(i=1,2, \cdots, 5)$ under the above four attributes $X=\left(x_{1}, x_{2}, x_{3}, x_{4}\right)$ on condition of anonymity. And suppose that the weight vector

Table 1: Interval-valued hesitant fuzzy decision matrix

\begin{tabular}{ccccc}
\hline & $x_{1}$ & $x_{2}$ & $x_{3}$ & $x_{4}$ \\
\hline$A_{1}$ & $\{[0.2,0.3],[0.3,0.4]\}$ & $\{[0.2,0.5]\}$ & $\{[0.7,0.8],[0.8,0.9]\}$ & $\{[0.4,0.5]\}$ \\
$A_{2}$ & $\{[0.2,0.3],[0.4,0.5],[0.5,0.6]\}$ & $\{[0.3,0.4],[0.6,0.7]\}$ & $\{[0.3,0.4]\}$ & $\{[0.5,0.6],[0.8,0.9]\}$ \\
$A_{3}$ & $\{[0.5,0.7]\}$ & $\{[0.2,0.3],[0.4,0.5]\}$ & $\{[0.8,0.9],[0.9,1.0]\}$ & $\{[0.3,0.5]\}$ \\
$A_{4}$ & $\{[0.3,0.4],[0.7,0.8]\}$ & $\{[0.1,0.3]\}$ & $\{[0.6,0.7],[0.8,0.9]\}$ & $\{[0.5,0.7]\}$ \\
$A_{5}$ & $\{[0.2,0.3]\}$ & $\{[0.4,0.6]\}$ & $\{[0.2,0.3],[0.6,0.7]\}$ & $\{[0.6,0.7]\}$ \\
\hline
\end{tabular}


of attributes is $(0.4,0.2,0.3,0.1)$. The decision matrix is presented in Table 1 below, which shows all interval-valued hesitant fuzzy information.

Step 1. Construct the corresponding interval- valued hesitant fuzzy sets according to the characteristics of the alternatives as follows based on Table 1:

$$
\begin{aligned}
A_{1}= & \left\{\left\langle x_{1},\{[0.2,0.3],[0.3,0.4]\}\right\rangle,\left\langle x_{2},\{[0.2,0.5]\}\right\rangle,\left\langle x_{3},\{[0.7,0.8],[0.8,0.9]\}\right\rangle,\left\langle x_{4},\{[0.4,0.5]\}\right\rangle\right\}, \\
A_{2}= & \left\{\left\langle x_{1},\{[0.2,0.3],[0.4,0.5],[0.5,0.6]\}\right\rangle,\left\langle x_{2},\{[0.3,0.4],[0.6,0.7]\}\right\rangle,\left\langle x_{3},\{[0.3,0.4]\}\right\rangle,\right. \\
& \left.\left\langle x_{4},\{[0.5,0.6],[0.8,0.9]\}\right\rangle\right\}, \\
A_{3}= & \left\{\left\langle x_{1},\{[0.5,0.7]\}\right\rangle,\left\langle x_{2},\{[0.2,0.3],[0.4,0.5]\}\right\rangle,\left\langle x_{3},\{[0.8,0.9],[0.9,1.0]\}\right\rangle,\left\langle x_{4},\{[0.3,0.5]\}\right\rangle\right\}, \\
A_{4}= & \left\{\left\langle x_{1},\{[0.3,0.4],[0.7,0.8]\}\right\rangle,\left\langle x_{2},\{[0.1,0.3]\}\right\rangle,\left\langle x_{3},\{[0.6,0.7],[0.8,0.9]\}\right\rangle,\left\langle x_{4},\{[0.5,0.7]\}\right\rangle\right\}, \\
A_{1}= & \left\{\left\langle x_{1},\{[0.2,0.3]\}\right\rangle,\left\langle x_{2},\{[0.4,0.6]\}\right\rangle,\left\langle x_{3},\{[0.2,0.3],[0.6,0.7]\}\right\rangle,\left\langle x_{4},\{[0.6,0.7]\}\right\rangle\right\} .
\end{aligned}
$$

Step 2. Calculate the positive ideal IVHFS and the negative ideal IVHFS as follows, respectively:

$$
\begin{aligned}
A^{+}= & \left\{\left\langle x_{1},\{[0.7,0.8]\}\right\rangle,\left\langle x_{2},\{[0.6,0.7]\}\right\rangle,\right. \\
& \left.\left\langle x_{3},\{[0.9,1.0]\}\right\rangle,\left\langle x_{4},\{[0.8,0.9]\}\right\rangle\right\}, \\
A^{-}= & \left\{\left\langle x_{1},\{[0.2,0.3]\}\right\rangle,\left\langle x_{2},\{[0.1,0.3]\}\right\rangle,\right. \\
& \left.\left\langle x_{3},\{[0.2,0.3]\}\right\rangle,\left\langle x_{4},\{[0.3,0.5]\}\right\rangle\right\} .
\end{aligned}
$$

For Step 3, we can utilize different similarity measures provided in Section 4 to determine the priority of the alternatives.

(Case 1) Utilizing the similarity measure $s_{1}^{\prime}$ with the weight vector $\omega$ for IVHFSs based on II-type generalized interval-valued hesitant fuzzy distance measures, i.e., Eq. (14).

Step 3. By (14), we calculate $s_{1}^{\prime}\left(A^{+}, A_{i}\right)$ and $s_{1}^{\prime}\left(A^{-}, A_{i}\right)(i=1,2,3,4,5)$ and assume that $p=2$.

$$
\begin{aligned}
& s_{1}^{\prime}\left(A^{+}, A_{1}\right)=0.6682, s_{1}^{\prime}\left(A^{+}, A_{2}\right)=0.6140, \\
& s_{1}^{\prime}\left(A^{+}, A_{3}\right)=0.8070, s_{1}^{\prime}\left(A^{+}, A_{4}\right)=0.7037, \\
& s_{1}^{\prime}\left(A^{+}, A_{5}\right)=0.5868 ; \\
& s_{1}^{\prime}\left(A^{-}, A_{1}\right)=0.7556, s_{1}^{\prime}\left(A^{-}, A_{2}\right)=0.7850, \\
& s_{1}^{\prime}\left(A^{-}, A_{3}\right)=0.6256, s_{1}^{\prime}\left(A^{-}, A_{4}\right)=0.6999, \\
& s_{1}^{\prime}\left(A^{-}, A_{5}\right)=0.8296 .
\end{aligned}
$$

Step 4. Calculate the relative similarity measure $r s_{i}$ corresponding to the alternative $A_{i}$ using Eq. (23)

$$
\begin{aligned}
& r s_{1}=0.4693, r s_{2}=0.4389, r s_{3}=0.5633, \\
& r s_{4}=0.5014, r s_{5}=0.4121 .
\end{aligned}
$$

Step 5. Obtain the priority of the alternative $A_{i}(i=1,2, \cdots, 5)$ by ranking the $r s_{i}(i=1,2, \cdots, 5)$.

$$
A_{3} \succ A_{4} \succ A_{1} \succ A_{2} \succ A_{5} \text {. }
$$

(Case 2) Utilizing the similarity measure $s_{2}^{\prime}$ with the weight vector $\omega$ for IVHFSs based on the interval-valued hesitant fuzzy $L_{p}$ distance.

Step 3. Calculate $s_{2}^{\prime}\left(A^{+}, A_{i}\right)$ and $s_{2}^{\prime}\left(A^{-}, A_{i}\right)(i=$ $1,2,3,4,5)$ and assume that $p=2$.

$$
\begin{aligned}
& s_{2}^{\prime}\left(A^{+}, A_{1}\right)=0.3969, s_{2}^{\prime}\left(A^{+}, A_{2}\right)=0.2695, \\
& s_{2}^{\prime}\left(A^{+}, A_{3}\right)=0.6776, s_{2}^{\prime}\left(A^{+}, A_{4}\right)=0.4754, \\
& s_{2}^{\prime}\left(A^{+}, A_{5}\right)=0.3210 ; \\
& s_{2}^{\prime}\left(A^{-}, A_{1}\right)=0.5573, s_{2}^{\prime}\left(A^{-}, A_{2}\right)=0.5501, \\
& s_{2}^{\prime}\left(A^{-}, A_{3}\right)=0.3340, s_{2}^{\prime}\left(A^{-}, A_{4}\right)=0.4116, \\
& s_{2}^{\prime}\left(A^{-}, A_{5}\right)=0.7094 .
\end{aligned}
$$

Step 4. Calculate the relative similarity measure $r s_{i}$ corresponding to the alternative $A_{i}$ using Eq. (23)

$$
\begin{aligned}
& r s_{1}=0.4159, r s_{2}=0.3288, r s_{3}=0.6698 \\
& r s_{4}=0.5360, r s_{5}=0.3115 .
\end{aligned}
$$

Step 5. Obtain the priority of the alternative $A_{i}(i=1,2, \cdots, 5)$ by ranking the $r s_{i}(i=1,2, \cdots, 5)$.

$$
A_{3} \succ A_{4} \succ A_{1} \succ A_{2} \succ A_{5} .
$$

(Case 3) Utilizing the similarity measure $s_{3}^{\prime}$ with the weight vector $\omega$ for IVHFSs based on the settheoretic approach. 
Step 3. Calculate $s_{3}^{\prime}\left(A^{+}, A_{i}\right)$ and $s_{3}^{\prime}\left(A^{-}, A_{i}\right)(i=$ $1,2,3,4,5)$.

$$
\begin{aligned}
& s_{3}^{\prime}\left(A^{+}, A_{1}\right)=0.2097, s_{3}^{\prime}\left(A^{+}, A_{2}\right)=0.2789, \\
& s_{3}^{\prime}\left(A^{+}, A_{3}\right)=0.3866, s_{3}^{\prime}\left(A^{+}, A_{4}\right)=0.2667, \\
& s_{3}^{\prime}\left(A^{+}, A_{5}\right)=0.2810 ; \\
& s_{3}^{\prime}\left(A^{-}, A_{1}\right)=0.5593, s_{3}^{\prime}\left(A^{-}, A_{2}\right)=0.5126, \\
& s_{3}^{\prime}\left(A^{-}, A_{3}\right)=0.3481, s_{3}^{\prime}\left(A^{-}, A_{4}\right)=0.5057, \\
& s_{3}^{\prime}\left(A^{-}, A_{5}\right)=0.65 .
\end{aligned}
$$

Step 4. Calculate the relative similarity measure $r s_{i}$ corresponding to the alternative $A_{i}$ using Eq. (23)

$$
\begin{aligned}
& r s_{1}=0.2727, r s_{2}=0.3523, r s_{3}=0.5262, \\
& r s_{4}=0.3453, r s_{5}=0.3018 .
\end{aligned}
$$

Step 5. Obtain the priority of the alternative $A_{i}(i=1,2, \cdots, 5)$ by ranking the $r s_{i}(i=1,2, \cdots, 5)$.

$$
A_{3} \succ A_{2} \succ A_{4} \succ A_{5} \succ A_{1} .
$$

According to the above numerical results for three cases utilizing different similarity measures under interval-valued hesitant fuzzy environment, the ordering of the alternatives are given in Eq. (24), Eq. (25), Eq. (26) respectively. The $\succ$ in Eqs. (24)-(26) means "preferred to". We can see from the results, depending on the similarity measures used, the ordering of the alternatives is slightly different. Therefore, depending on the similarity measure used, the result will lead to different ranking of other alternatives, but the best desirable alternative is the same. From Eq. (24) to Eq. (26), we know that alternative $A_{3}$ is the best one obtained by using all three similarity measures proposed in Section 4.

In addition, in ${ }^{22}$, Wei. et al. applied an intervalvalued hesitant fuzzy choquet ordered averaging (IVHFCOA) operator and a hesitant interval-valued fuzzy choquet ordered geometric (IVHFCOG) operator to the same practical example with intervalvalued hesitant fuzzy information. As the results of aggregation are some intervals, so, a score function of IVFHNs was employed in order to obtain the ordering of the alternatives. The result is the same as the one obtained using the proposed method based on the relative similarity measures for IVHFSs. This shows the proposed method is rational and practically feasible.

\section{Conclusions}

IVHFSs, which is a generalization of the HFSs, have been used widely in decision problems ${ }^{17,19}$. IVHFS permits the memberships of an element to a given set having a few different interval values in $[0,1]$ rather than precise single numbers, so it can be considered as a useful tool to express uncertain information in the human decision making process. In this paper, our aim mainly focused on the following two aspects:

(1) New information measure A generalization of popular Haming-distance (that is, a II-type generalized interval-valued hesitant fuzzy distance) and an interval-valued hesitant fuzzy $L p$ distance were introduced based on some existing distance measures for IVHFSs and $L_{p}$ metric, the relation between interval-valued hesitant fuzzy $L_{p}$ distance and interval-valued hesitant fuzzy Hausdorff distance was also discussed. About the similarity measure, we analysed the existing similarity measures, a family of similarity measures for IVHFSs were obtained and a new similarity measure, which is not dependent on the distance measure in IVHFSs from the view of set theory, was proposed.

(2) Application of relative similarity measure We proposed the concept of relative similarity measure of IVHFSs based on the new similarity measure in (1) and constructed a multiple attribute decision making approach under interval-valued hesitant fuzzy environment and based on the relative similarity measure. Consequently, a practical example was provided to illustrate this method and compare it with the existing one $i^{22}$.

In the future, we shall continue working applying the interval-valued hesitant fuzzy multiple attribute decision making approach to other problem domains, and also focus on other information measures on hesitant fuzzy set and its application in the real world. 


\section{Acknowledgments}

The authors are grateful to the anonymous reviewers for their constructive comments and based on which the presentation of this paper has been greatly improved. This work is partially supported by the National Natural Science Foundation of P.R.China (Grant no. 61673320, 61305074); Chinese Scholarship Council of the Ministry of Education ([2016]5112); the Application Basic Research Plan Project of Sichuan Province (No.2015JY0120); the Scientific Research Project of Department of Education of Sichuan Province (15TD0027, 14ZA0245, 15ZB0270); Natural Science Foundation of Guangdong Province (2016A030310003).

\section{References}

1. K. Atanassov, G. Gargov, Interval-valued intuitionistic fuzzy sets, Fuzzy Sets and Systems, 31 (1989) 343C349.

2. K. Atanassov, Operators over interval-valued intuitionistic fuzzy sets, Fuzzy Sets and Systems, 64 (1994) 159C174.

3. K.T. Attanassov, Intuitionistic fuzzy sets, Fuzzy Sets and Systems, 20 (1986) 87-96.

4. S-J. Chen, C-L. Hwang, Fuzzy multiple attribute decision making: methods and application, Springerverlag, Berlin, 1992.

5. N. Chen, Z. Xu, M. Xia, Interval-valued hesitant preference relations and their applications to group decision making,Knowledge-Based Systems, 37 (2013) 528-540.

6. N. Chen, Z.S. Xu, Properties of interval-valued hesitant fuzzy sets, Journal of Intelligent and Fuzzy Systems, 27 (2014) 143-158.

7. B. Farhadinia, Information measures for hesitant fuzzy sets and interval-valued hesitant fuzzy sets, Information Sciences, 240 (2013) 129-144.

8. B. Farhadinia, Correlation for Dual Hesitant Fuzzy Sets and Dual Interval-Valued Hesitant Fuzzy Sets, International Journal of Intelligent Systems, 29 (2014) 184-205.

9. B. Farhadinia, Study on division and subtraction operations for hesitant fuzzy sets, interval-valued hesitant fuzzy sets and typical dual hesitant fuzzy sets, Journal of Intelligent Fuzzy Systems, 28 (2015) 1393-1402.

10. H. Gitinavard, S.M. Mousavi, B. Vahdani, A new multi-criteria weighting and ranking model for group decision-making analysis based on interval-valued hesitant fuzzy sets to selection problems, Neural Comput. Appl., 27 (2016) 1593-1605.

11. Y. Liu, Y. Qin, Y. Han, Multiple criteria decision making with probabilities in interval- valued Pythagorean fuzzy setting, International Journal of Fuzzy System, DOI 10.1007/s40815-017-0349-3.

12. W.-L. Hung, M.-S. Yang, Similarity measures of intuitionistic fuzzy sets based on Lp metric, International Journal of Approximate Reasoning, 46 (2007) 120-136.

13. F. Jin, Z. Ni, H. Chen, Y. Li, L. Zhou, Multiple attribute group decision making based on intervalvalued hesitant fuzzy information measures, Computers Industrial Engineering, 101 (2016) 103-115.

14. D. Joshi, S. Kumar, Interval-valued intuitionistic hesitant fuzzy Choquet integral based TOPSIS method for multi-criteria group decision making, European Journal of Operational Research, 248 (2016) 183-191.

15. X. Peng, Y. Yang, Fundamental properties of intervalValued Pythagorean fuzzy aggregation operators. Journal of Intelligent Fuzzy Systems, 31(2016)444487.

16. P. Quirs, P. Alonso, H. Bustince, I. Diaz, S. Montes, An entropy measure definition for finite intervalvalued hesitant fuzzy sets, Knowledge-Based Systems, 84 (2015) 121-133.

17. R.M. Rodriguez, B. Bedregal, H. Bustince, Y.C. Dong, B. Farhadinia, C. Kahraman, L. Martinez, V. Torra, Y.J. Xu, Z.S. Xu, F. Herrera, A position and perspective analysis of hesitant fuzzy sets on information fusion in decision making. Towards high quality progress, Information Fusion, 29 (2016) 89-97.

18. Rosa M. Rodrłguez, lvaro Labella, Luis Martłnez, An Overview on Fuzzy Modelling of Complex Linguistic Preferences in Decision Making, International Journal of Computational Intelligence Systems, 9 (2016)81-94

19. R.M. Rodriguez, L. Martinez, V. Torra, Z.S. Xu, F. Herrera, Hesitant Fuzzy Sets: State of the Art and Future Directions, International Journal of Intelligent Systems, 29 (2014) 495-524.

20. V. Torra, Hesitant fuzzy sets, International Journal of Intelligent Systems, 25 (2010) 529-539.

21. G.W. Wei, R. Lin, H.J. Wang, Distance and similarity measures for hesitant interval-valued fuzzy sets, Journal of Intelligent Fuzzy Systems, 27 (2014) 19-36.

22. G.W. Wei, X.F. Zhao, R. Lin, Some hesitant intervalvalued fuzzy aggregation operators and their applications to multiple attribute decision making, Knowledge-Based Systems, 46 (2013) 43-53.

23. L.A. Zadeh, Fuzzy sets, Information and Computation, 8 (1965) 338-353. 